\title{
Acoustic Waves in Bubbly Soft Media
}

\author{
Bin Liang1, Ying Yuan², Xin-ye Zou ${ }^{1}$ and Jian-chun Cheng1 \\ ${ }^{1}$ Nanjing University, Nanjing \\ ${ }^{2}$ Jiangsu Teachers University of Techonology, Changzhou \\ P. R. China
}

\section{Introduction}

There exists a special class of solid media called soft media (or weakly compressible media), for which the inequality $\lambda>>\mu$ is satisfied ( $\lambda$ and $\mu$ are the Lamé coefficients) [1-6]. Such media with very small shear stiffness are dynamically similar to liquids to a great extent and exhibit strongly a "water-like" characteristic and, therefore, are also associated with the name of "water-like" media. The class of soft media includes many common media in the fields of scientific research and practical applications (e.g. soft rubbers, tissues, or biomimetic materials), and air bubbles are often introduced due to artificial or non-artificial reasons. In the presence of air bubbles in a soft medium, the bubbles will oscillate violently as an acoustic wave propagates in the bubbly medium. It has been proved that only if the ratio $\lambda>>\mu$ is sufficiently large can a bubble in a solid behave like an oscillator with a large quality factor, otherwise oscillation will be damped within a short time comparable to its period [7]. This results in the fact that the acoustical property of a bubbly soft medium is particularly different from that of a usual solid medium containing bubbles. The strong scattering of acoustic waves by bubbles in soft media may lead to some significant physical phenomena stemming from the multiple-scattering effects, such as the localization phenomenon [8-11]. On the other hands, a bubbly soft medium has potential applications to a variety of important situations, such as the fabrication of acoustic absorbent with high efficiency or the clinic application of ultrasonic imaging utilizing the contrast agents [12]. Consequently, it is of academic as well as practical significance to make a comprehensive study on the acoustic waves in a bubbly soft medium.

Attempts to theoretically investigate the bubble dynamics in a soft medium go back many decades [1-4]. Meyer et al have performed the early measurements of the resonance frequency of a bubble in rubbers [1]. The dynamical equation for arbitrarily large radial motion has been derived by Erigen and Suhubi in Rayleigh-Plesset form [2]. Ostrovsky has derived a Rayleigh-Plesset-like equation to describe the nonlinear oscillation of an individual bubble in a soft medium [3,4]. On the basis of the linear solution of the bubble dynamic equation of Ostrovsky, Liang and Cheng have theoretically investigated the acoustic propagation in an elastic soft medium containing a finite number of bubbles [8-11]. By rigorously solving the wave field using a multiple-scattering method, they have revealed the ubiquitous existence of the significant phenomenon of acoustic localization in such a class of media, and identified the "phase transition" phenomenon similar with the orderdisorder phase transition in a ferromagnet. For practical samples of bubbly soft media, however, the multiple-scattering method usually fails due to the extremely large number of 
bubbles and the strong acoustical nonlinearity in such media. In such cases, a more useful approach is to treat the bubbly soft media as a homogeneous "effective" medium characterized by nonlinear effective parameters, which are remarkably influenced by many factors associated with bubble oscillations such as surface tension, compressibility, viscosity, surrounding pressure, and an encapsulating elastic shell. Emelianov et al have studied the influence of surrounding pressure on the nonlinear dynamics of a bubble in an incompressible medium [5]. Their model has then been extended by Zabolotskaya et al to account for the effects of surface tension, viscosity, weak compressibility, and elastic shell [6]. Based on the bubble dynamics model of Zabolotskaya et al, Liang et al have developed an effective medium method (EMM) to give description of the nonlinear sound propagation in bubbly soft media with these effects accurately included [12]. Compared with the viscosity of soft medium, the resonance of the system introduced by bubbles becomes the most dominant mechanism for acoustic attenuation in a bubbly soft medium which necessarily depends on the parameters of bubbles [13]. With the availability of obtaining the effective acoustical parameters of bubbly soft media, Liang et al have presented an optimization method on the basis of fuzzy logic and genetic algorithm to yield the optimal acoustic attenuation of such media by optimizing the parameters of the size distribution of bubbles [14].

The chapter is structured as follows: we first focus on the phenomenon of acoustic localization in elastic soft media containing finite numbers of bubbles in Section 2. In Section 3 , we study the phase transition in acoustic localization which helps to identify the phenomenon of localization in the presence of viscosity. In Section 4, we discuss the EMM to describe nonlinear acoustic property of bubbly soft media. Finally in Section 5, we study how to enhance the acoustic attenuation in soft bubbly media in an optimal manner.

\section{Acoustic localization in bubbly soft media}

In this section, we theoretically study the sound propagation in an elastic soft medium containing a finite number of bubbles by using a self-consistent method to reveal the existence of acoustic localization under proper conditions. It will be proved that the phenomenon of localization can be identified by properly analyzing the spatial correlation of wave field.

\subsection{The model}

Consider a longitudinal wave in an elastic soft medium containing air bubbles with small volume fraction $\beta$. We shall restrict our attention to the wave propagation at low frequencies, i.e., $k r_{0}<<1$. Here $k=\omega / c_{l}$ and $c_{l}=\sqrt{(\lambda+2 \mu) / \rho}$ are the wavenumber and the speed of the longitudinal wave, respectively, and $\rho$ is the mass density. The wave is assumed to be of angular frequency $\omega$ and emitted from a unit point source located at the origin, surrounded by $N$ spherical air bubbles that are randomly located at $\mathbf{r}_{i}$ in a not overlapping way with $i=1,2, \cdots, N$. For simplicity and without loss of generality, all the bubbles are assumed to be of uniform radius $r_{0}$ and randomly distributed within a spatial domain, which is taken as the spherical shape so as to eliminate irrelevant effects due to an irregular edge. Then the radius of the spherical bubble cloud is $R_{0}=(N / \beta)^{1 / 3} r_{0}$. Here the source is placed inside rather than outside the bubble cloud, which is the only way to isolate the localization effect from boundary effects and unambiguously investigate the problem of whether the transmitted waves can indeed be trapped [15]. 


\subsection{Validation of approximation}

As the incident longitudinal wave is scattered by a bubble in soft media, the energy converted into shear wave can be expected negligible, on condition of sufficiently small shear stiffness. Next we shall present a numerical demonstration of the validation of such an approximation by inspecting the scattering cross section of a single bubble in soft media.

The total scattering cross section $\sigma$ of a single bubble can be expressed as $\sigma=\sigma_{L}+\sigma_{S}$, where $\sigma_{L}$ and $\sigma_{S}$ refer to the contributions of the scattered longitudinal and shear waves, respectively. Therefore it is conceivable that the ratio $\sigma_{L} / \sigma_{S}$ actually reflects the extent to which the mode conversion will occur as the incident longitudinal wave is scattered by the bubble. The value of $\sigma_{L} / \sigma_{S}$ can be readily obtained from Eq. (19) in Ref. [16].

A series of numerical experiments has been performed for an individual bubble in a variety of media, for the purpose of investigating how the energy converted into shear wave is affected as the media of matrix varies. Figure 2.1 presents the typical result of the comparison between the ratios $\sigma_{L} / \sigma_{S}$ versus frequency $k r_{0}$ for different media of matrix. Three particular kinds of elastic soft media are considered: Agar-gelatin, plastisol, and gelatin. Aluminum is also considered for comparison. The physical parameters of these materials are listed in Table 2.1. Note that the physical parameters of soft media approximate to those of water except for different values of shear moduli, which seems to be natural for such "waterlike" media.

From Fig. 2.1, we find: (1) the energy of the scattered shear wave is extremely small when compared to that of the scattered longitudinal wave, as long as the ratios $\lambda / \mu$ are sufficiently large. Contrarily the magnitude of the longitudinal components is nearly the same to the shear components in the scattered field for $\mu$ comparable with $\lambda$. (2) for an individual bubble in different soft media, the energy converted into shear wave is diminished as the shear modulus of the medium of matrix decreases, as expected. (3) moreover, the ratios $\sigma_{L} / \sigma_{S}$ are greatly enhanced in the proximity of the natural resonance of the individual bubbles, owing to the giant monopole resonance that is the dominant mode of bubble pulsation at low frequencies [17].

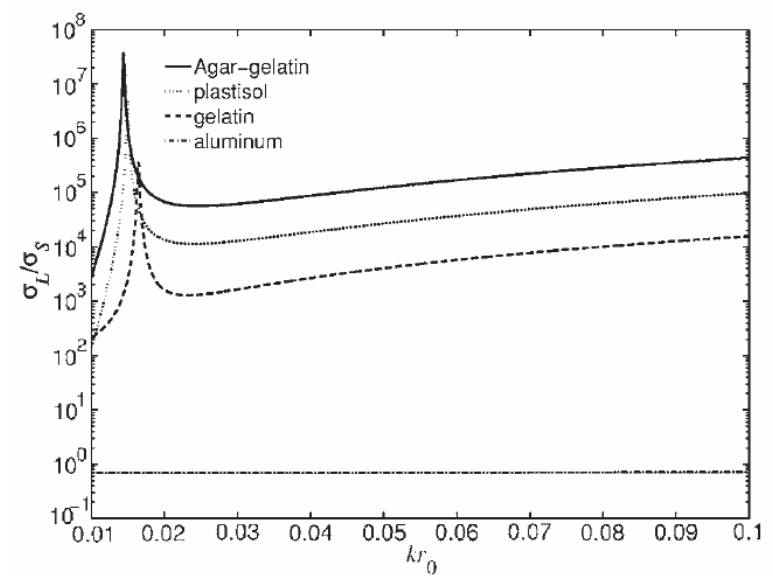

Fig. 2.1 The ratio $\sigma_{L} / \sigma_{S}$ versus frequency $k r_{0}$ for a single bubble in different materials. 
Thereby it follows that the energy converted into shear waves is insignificant as the incident longitudinal wave is scattered by a bubble in soft media. The shear component of the scattered field may thus be expected to be negligible as the longitudinal wave propagates in a bubbly soft medium. It should be stressed, however, that such an approximation can only be regard valid under the condition that the propagation of the elastic waves is not completely diffusive. Otherwise there will be an entirely contradicting conclusion that the ratio between the energy of the shear and the longitudinal wave is as large as $2\left(c_{l} / c_{s}\right)^{3}$ when the wave field is completely diffusive $[18,19]$. Here $c_{s}=\sqrt{2 \mu / \rho}$ is the speed of the shear wave.

Such a seeming paradox may be clarified by estimating the ratio between the mean free path (MFP) of the wave and the linear "sample size" $R$ of the random medium. For a classical wave propagating in a random media, the transport MFP is defined as the length over which momentum transfer becomes uncorrelated, while the elastic MFP is the average distance between two successive scattering events [20].

For an elastic wave, the following inequality has to be fulfilled to completely attain the diffusive regime [21]:

$$
1 / k<<l_{T_{l}}<<R, 1 / \kappa<<l_{T_{s}}<<R,
$$

where $l_{T_{l}}$ and $l_{T_{s}}$ refer to the transport MFPs of longitudinal wave and shear wave respectively, $\kappa=\omega / c_{s}$ is the wave number of the shear wave. Otherwise the wave propagation will be predominantly ballistic as the ratio between the MFP and the sample size is large [22].

At low frequencies from $k r_{0}=0$ to 0.1 , a set of numerical experiments have been carried out to estimate the MFPs of the elastic waves in various bubbly soft media for all the structure parameters employed in the present study. The typical values of the sample size $R$ lie roughly within the range $4 \times 10^{-4} \mathrm{~m} \leq R \leq 0.8 \mathrm{~m}$. The results indicate that: (1) for the shear wave in bubbly soft media, the typical values of the transport MFP $l_{T_{s}}$ and the elastic MFP $l_{E_{s}}$ of shear wave lie approximately within the range $6 \times 10^{-3} \mathrm{~m} \leq l_{T_{s}}, l_{E_{s}} \leq 4 \times 10^{5} \mathrm{~m}$. For any particular set of structure parameters, both the transport MFP $l_{T_{s}}$ and the elastic MFP $l_{E_{s}}$ are much larger than the sample size, i.e., $l_{E_{s}}, l_{T_{s}} \gg R$. This manifests that the scattering of the low-frequency shear wave by the bubbles in a soft medium is weak and, therefore, the propagation of the shear wave in such a medium is ballistic rather than diffusive. (2) for the longitudinal wave, for most frequencies the range of the typical values of $l_{T_{l}}$ and $l_{E_{l}}$ are roughly $4 \times 10^{-3} \mathrm{~m} \leq l_{T_{l}}, l_{E_{l}} \leq 3 \times 10^{5} \mathrm{~m}$ and the relation $l_{E_{l}}, l_{T_{l}} \gg R$ holds for any particular set of structure parameters, except for a frequency range within which $1 \times 10^{-6} \mathrm{~m} \leq l_{T_{l}} \leq 0.8 \mathrm{~m}$ and the Ioffe-Regel criterion $k l_{T_{l}} \leq 1$ is satisfied for any particular set of structure parameters [23]. As will be explained later, such a frequency range is in fact the region where the phenomenon of acoustic localization occurs. This is consistent with the conclusion regarding a bubbly liquid that the coherent wave dominates the transmission for most frequencies except for the localization region [24].

As a result, the condition to attain a completely diffusive wave field can not be satisfied at low frequencies as the longitudinal wave propagates in a bubbly soft medium. Consequently, the shear component of the scattered field is unimportant when compared to the longitudinal component, and neglect of mode conversion only leads to negligible errors. 
The results of the numerical experiments indicate that the numerical error caused by neglecting mode conversion is less than $1 \%$ as long as the ratio $\lambda / \mu$ is roughly larger than $10^{4}$. In the following study, we shall assume the scattered field to be totally longitudinal without taking mode conversion into consideration.

\begin{tabular}{|c|c|c|c|c|}
\hline Materials & $\rho\left(\mathrm{kg} / \mathrm{m}^{3}\right)$ & $\lambda$ & $\mu$ & $\lambda / \mu$ \\
\hline Agar-gelatin $^{\mathrm{a}}$ & 1000 & $2.25 \mathrm{GPa}$ & $6.35 \mathrm{KPa}$ & $4 \times 10^{5}$ \\
\hline plastisol $^{\mathrm{b}}$ & 1000 & $2.55 \mathrm{GPa}$ & $12.1 \mathrm{KPa}$ & $2 \times 10^{5}$ \\
\hline gelatinc $^{\mathrm{a}}$ & 1000 & $2.25 \mathrm{GPa}$ & $39.0 \mathrm{KPa}$ & $5 \times 10^{4}$ \\
\hline aluminum $^{2}$ & 2700 & $111.3 \mathrm{GPa}$ & $37.1 \mathrm{GPa}$ & 3 \\
\hline
\end{tabular}

aReference [25].

bReferences [4], [26], and [27].

cReference [6].

Table 2.1. The physical parameters of the materials

\subsection{The bubble dynamics}

For an individual bubble in soft elastic media, the radial pulsation driven by a plane longitudinal wave is described by a Rayleigh-Plesset-like equation, as follows $[3,4]$ :

$$
\frac{d^{2} U}{d t^{2}}+\omega_{0}^{2} U-\frac{r_{0}}{c_{l}} \frac{d^{3} U}{d t^{3}}=G U^{2}+q\left[2 U \frac{d^{2} U}{d t^{2}}+\left(\frac{d U}{d t}\right)^{2}\right]-e p_{\text {inc }},
$$

where $U=4 \pi\left(r_{i n}^{3}-r_{0}^{3}\right) / 3$ is volume perturbation of an individual bubble with $r_{\text {in }}$ being the instantaneous radius, $p_{\text {inc }}$ refers to the incident plane wave on the bubble, i.e., the driving force of the bubble pulsation, $\omega_{0}=\sqrt{\omega_{1}^{2}+\omega_{2}^{2}}$ is the bubble resonance frequency with $\omega_{1}=\sqrt{4 \mu / \rho r_{0}^{2}}$ and $\omega_{2}=\sqrt{3 \rho_{a} c_{a}^{2} / \rho r_{0}^{2}}$ corresponding to the Meyer-Brendel-Tamm resonance and the Minnaert bubble resonance, respectively [8], the parameters of $G, q$, and $e$ are given as $G=(9+2 \chi) q \omega_{0}^{2} / 2, q=1 /\left(8 \pi r_{0}^{3}\right), e=4 \pi r_{0} / \rho$. Here $\chi$ is a coefficient of asymmetry, which must lie in the range $0<\chi \leq 1$. For a spherical bubble $\chi=1$. Up to the first order approximation, one obtains the harmonic solution of Eq. (2.1), as follows

$$
U=-\frac{4 \pi r_{0}}{\rho\left(\omega_{0}^{2}-\omega^{2}-i \omega^{3} r_{0} / c_{l}\right)} p_{i n c}
$$

At low frequencies, the acoustic field produced by acoustic radiation from a single bubble is taken to be the diverging spherical wave [6]

$$
p_{s}(\mathbf{r}, t)=p_{s}(\mathbf{r}) \exp (-i \omega t) \approx-\frac{1}{4 \pi r} \frac{d^{2}}{d t^{2}} U\left(t-\frac{r}{c_{l}}\right),
$$

We assume that the bubble is the $i$ th bubble located at $\mathbf{r}_{i}$ and express the incident plane wave as

$$
p_{\text {inc }}(\mathbf{r}, t)=p_{\text {inc }}(\mathbf{r}) \exp (-i \omega t)
$$




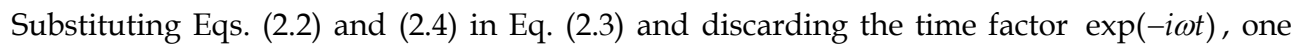
obtains the scattered wave at $\mathbf{r}$ from the $i$ th bubble, denoted by $p_{s}^{i}(\mathbf{r})$, as follows:

$$
p_{s}^{i}(\mathbf{r})=f p_{\text {inc }}\left(\mathbf{r}_{i}\right) G_{0}\left(\mathbf{r}-\mathbf{r}_{i}\right),
$$

where $G_{0}\left(\mathbf{r}-\mathbf{r}_{i}\right)=\exp \left(i k\left|\mathbf{r}-\mathbf{r}_{i}\right|\right) /\left|\mathbf{r}-\mathbf{r}_{i}\right|$ is the usual three-dimensional Green's function, $f$ is the scattering function of a single bubble, defined as

$$
f=\frac{r_{0}}{\left(\omega_{0}^{2} / \omega^{2}-1-i k r_{0}\right)}
$$

Eqs. (2.5) and (2.6) clearly show that the scattered fields and scattering function of a single bubble in soft media take an identical form as in liquid media, except for different expressions of $\omega_{0}$ (see Eqs. (2) and (2) in Ref. [24] and also Eq. (2) in Ref. [28]). Compared with the resonance frequency of a bubble in liquid media that includes only the Minnaert contribution $\omega_{2}$, an additional term $\omega_{1}$ is present in Eq. (2.6), due to the contribution of shear wave in the solid wall of bubble. In the limiting case $\lambda / \mu \rightarrow \infty$, the soft medium becomes a liquid medium, and Eq. (2.6) degenerates to Eq. (4) in Ref. [24] due to the vanishing of $\omega_{1}$. Despite such an agreement of the results in the form, we have to particularly stress that it is the radial pulsation equation of a single bubble from which the present theory begins, rather than the well-studied scattering function of a bubble employed in Ref. [24].

\subsection{The self-consistent formalism}

Among many useful formalisms suggested for describing the acoustic scattering by a finite group of random scatterers, the self-consistent method is proved to be particularly effective [29]. This method is based on a genuine self-consistent scheme proposed first by Foldy [30] and reviewed in Ref. [31]. Later Ye et al employed the self-consistent method to investigate the wave propagation in a bubble liquid [24]. In the method, the multiple scattering of waves is represented by a set of coupled equations, and the rigorous results can be obtained by solving the equations. In the present study, the wave propagation in a bubbly soft medium will be solved by using the self-consistent method in an exact manner.

In the presence of bubbles, the radiated wave from the source is subject to multiple scattering by the surrounding bubbles. Hence the total acoustic wave at a spatial point $\mathbf{r}$ is supposed to include the contributions from the wave directed from the source and the scattered waves from all bubbles, i.e.,

$$
p(\mathbf{r})=p_{0}(\mathbf{r})+\sum_{i=1}^{N} p_{s}^{i}(\mathbf{r}),
$$

Analogously, the incident wave upon the $i$ th bubble should consist of the direct wave from the source and the scattered waves from all bubbles except for itself, i.e., [31]

$$
p_{\text {inc }}\left(\mathbf{r}_{i}\right)=p_{0}\left(\mathbf{r}_{i}\right)+\sum_{j=1, j \neq i}^{N} p_{s}^{j}\left(\mathbf{r}_{i}\right),
$$


At $k r_{0}<<1$, the dimension of bubbles is very small when compared to the wavelength, and the plane wave approximation still stands for the incident wave upon an individual bubble. In this situation, the dynamics of an individual bubble can be described by the radial pulsation equation given by Eq. (2.1). Therefore for the $i$ th bubble, according to Eqs. (2.5) and (2.8), the following self-consistent equation should be satisfied:

$$
p_{s}^{i}(\mathbf{r})=f\left[p_{0}\left(\mathbf{r}_{i}\right)+\sum_{j=1, j \neq i}^{N} p_{s}^{j}\left(\mathbf{r}_{i}\right)\right] G_{0}\left(\mathbf{r}-\mathbf{r}_{i}\right),
$$

Upon incidence, each bubble acts effectively as a secondary pulsating source. The scattered wave from the $i$ th bubble is regarded as the radiated wave and is rewritten as [28]

$$
p_{s}^{i}(\mathbf{r})=A_{i} G_{0}\left(\mathbf{r}-\mathbf{r}_{i}\right)
$$

where the complex coefficient $A_{i}$ refers to the effective strength of the secondary source. Substitution of Eq. (2.10) in Eq. (2.8) yields

$$
A_{i}=f\left[p_{0}\left(\mathbf{r}_{i}\right)+\sum_{j=1, j \neq i}^{N} A_{j} G_{0}\left(\mathbf{r}_{j}-\mathbf{r}_{i}\right)\right],
$$

By setting $\mathbf{r}$ in Eq. (2.10) to any bubble other than the $i$ th, this equation becomes a set of closed self-consistent equations which can be expressed as

$$
\mathbf{A}=f \mathbf{P}+\mathbf{C A}
$$

where $\mathbf{A}=\left[A_{1}, A_{2}, \ldots, A_{N}\right]^{T}, \mathbf{P}=\left[p_{0}\left(\mathbf{r}_{1}\right), p_{0}\left(\mathbf{r}_{2}\right), \ldots, p_{0}\left(\mathbf{r}_{N}\right)\right]^{T}, \mathbf{C}$ is an $N \times N$ matrix, defined as

$$
\mathbf{C}=\left[\begin{array}{ccc}
C_{11} & \cdots & C_{1 N} \\
\vdots & \ddots & \vdots \\
C_{N 1} & \cdots & C_{N N}
\end{array}\right]
$$

where $C_{i j}=\left(1-\delta_{i j}\right) f G_{0}\left(\mathbf{r}_{j}-\mathbf{r}_{i}\right)$ with $\delta_{i j}$ being the Kronecker symbol. It is apparent that $\mathbf{C}$ is symmetric according to the principle of reciprocity. One may readily obtain from Eq. (2.12)

$$
\mathbf{A}=f(1-\mathbf{C})^{-1} \mathbf{P}
$$

For an arbitrary configuration of the bubble distribution, the acoustic field in any spatial point may thus be solved exactly from Eqs. (2.7), (2.10) and (2.13). It is obvious that the multiple scattering effects have been incorporated during the computation [24].

\subsection{The spatial correlation function}

With the purpose of identifying efficiently the phenomenon of localization, we consider the spatial correlation that physically describes the interaction between the wave fields at different spatial points. For an arbitrary spatial point $\mathbf{r}$, the wave field is normalized as $T(r)=p(r) / p_{0}(r)$ to eliminate the uninteresting geometrical spreading factor. Here $r=|\mathbf{r}|$ is the distance from the source point to the spatial point, $p(r)$ refers to the acoustic pressure averaged over the sphere of radius $r$, and $p_{0}(r)=\exp (i k r) / r$ refers to the propagating wave radiated from the source in the absence of bubbles. 


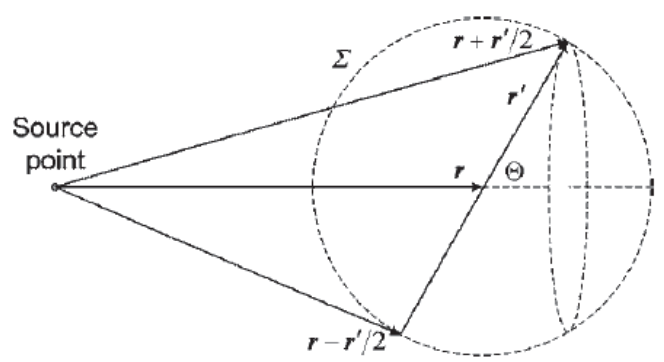

Fig. 2.2. Geometry for spatial correlation function of wave fields at $\mathbf{r}+\mathbf{r}^{\prime} / 2$ and $\mathbf{r}-\mathbf{r}^{\prime} / 2$.

Consider the interaction between the two spatial points $\mathbf{r}+\mathbf{r}^{\prime} / 2$ and $\mathbf{r}-\mathbf{r}^{\prime} / 2$, as shown in Fig. 2.2. The spatial correlation is defined as the average over the sphere $\Sigma$ that is located at $\mathbf{r}$ and of radius $\xi / 2$. Here $\xi=\left|\mathbf{r}^{\prime}\right|$ is the distance between $\mathbf{r}+\mathbf{r}^{\prime} / 2$ and $\mathbf{r}-\mathbf{r}^{\prime} / 2$. Note that the normalized wave field $T(r)$ is axially symmetric about $\mathbf{r}$ and depends only on $\Theta$. The average can thus be accomplished by performing the integration with respect to $\Theta$. Then the spatial correlation function is expressed as follows:

$$
\begin{array}{r}
g\left(\mathbf{r}+\boldsymbol{r}^{\prime} / 2, \mathbf{r}-\boldsymbol{r}^{\prime} / 2\right)=\frac{\int_{0}^{\pi} 2 \pi\left\langle T\left(\left|\mathbf{r}+\boldsymbol{r}^{\prime} / 2\right|\right) T^{*}\left(\left|\mathbf{r}-\boldsymbol{r}^{\prime} / 2\right|\right)\right\rangle \cdot(\xi / 2)^{2} \sin \Theta d \Theta}{4 \pi(\xi / 2)^{2}} \\
=\frac{1}{2} \int_{0}^{\pi}\left\langle T\left(\left|\mathbf{r}+\boldsymbol{r}^{\prime} / 2\right|\right) T^{*}\left(\left|\mathbf{r}-\boldsymbol{r}^{\prime} / 2\right|\right)\right\rangle \sin \Theta d \Theta
\end{array}
$$

where $\left|\mathbf{r} \pm \boldsymbol{r}^{\prime} / 2\right|=\sqrt{r^{2}+\xi^{2} / 4 \pm \xi r \cos \Theta}$, and $\langle\cdot\rangle$ refers to the ensemble average carried over random configuration of bubble clouds.

It is apparent that the preceding definition of the spatial correlation function refers to the average interaction between the wave fields at every pair of spatial points for which the distance is $\xi$ and the center of symmetry locates at $\mathbf{r}$. By using Eq. (2.14) and taking the ensemble average over the whole bubble cloud, then, we define the total correlation function that is a function of the distance $\xi$ so as to describe the overall correlation characteristics of the wave field. In respect that the normalized wave field $T(r)$ is symmetric about the origin, the total correlation function can be obtained by merely performing the integration with respect to $r$, given as below:

$$
C(\xi)=\frac{\int_{0}^{R_{0}} 4 \pi r^{2} g\left(\mathbf{r}+\boldsymbol{r}^{\prime} / 2, \mathbf{r}-\boldsymbol{r}^{\prime} / 2\right) d r}{\int_{0}^{R_{0}} 4 \pi r^{2} g(\mathbf{r}, \mathbf{r}) d r} .
$$

\subsection{Acoustic localization in bubbly elastic soft media}

A set of numerical experiments has been carried out for various bubble radii, numbers and volume fractions. Figure 2.3 presents the typical results of the total transmission and the total backscattering versus frequency $k r_{0}$ for bubbly gelatin with the parameters $N=200$, $r_{0}=1 \mathrm{~mm}$, and $\beta=10^{-3}$, respectively. The total transmission is defined as $I=\left\langle|T|^{2}\right\rangle$, and 
the received point is located at the distance $r=2 R$ from the source. The total backscattering is defined as $\left\langle\left|\sum_{i}^{N} p_{s}^{i}(0)\right|^{2}\right\rangle$, referring to the signal received at the transmitting source.

It is clearly suggested in Fig. 2.3(a) that there is a region of frequency slightly above the bubble resonance frequency, i.e., approximately between $k r_{0}=0.017$ and 0.077 in this particular case, in which the transmission is virtually forbidden. Within this frequencies range, the Ioffe-Regel criterion is satisfied and a maximal decrease of the diffusion coefficient $D$ roughly by a factor of $10^{5}$ is observed and $D$ can thus be considered having a tendency to vanish, i.e., $D \rightarrow 0$. Here the diffusion coefficient is defined as $D=v_{t} l_{T_{l}} / 3$ with $v_{t}$ being the transport velocity that may be estimated by using an effective medium method [32]. Indeed, this is the range that suggests the acoustic localization where the waves are considered trapped [24], confirming the conjectured existence of the phenomenon of localization in such a class of media. Outside this region, wave propagation remains extended. For the backscattering situation, the result shows that the backscattering signal persists for all the frequencies, and an enhancement of backscattering occurs particularly in the localization region. As has been suggested by Ye et al, however, the backscattering enhancement that appears as long as there is multiple scattering can not act as a direct indicator of the phenomenon of localization [28]. In the following we shall thus focus our attention on the transmission that helps us to identify the localization regions, rather than the backscattering of the propagating wave.
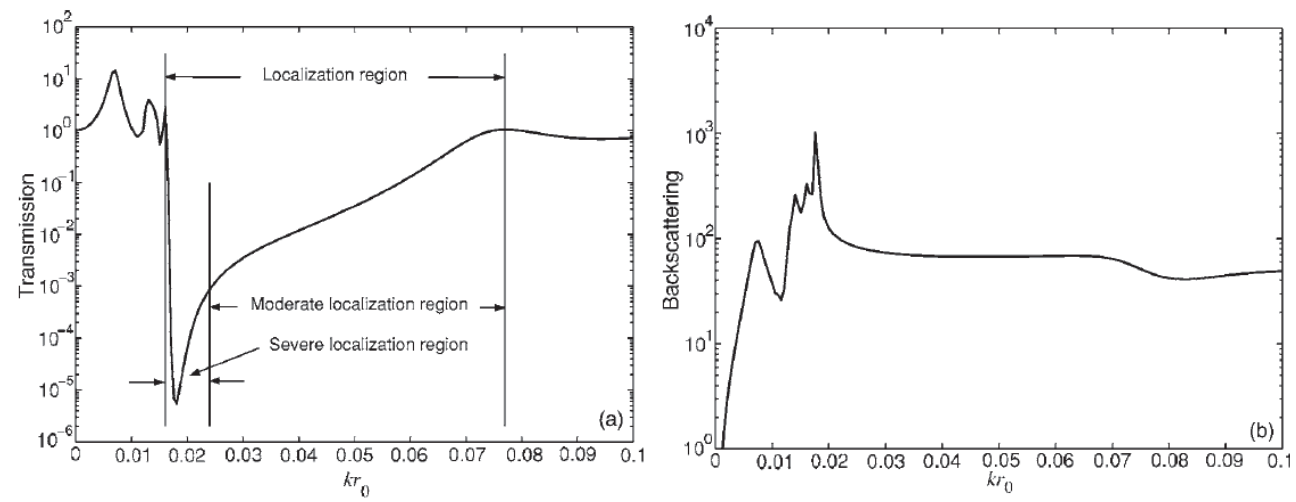

Fig. 2.3. The total transmission (a) and the total backscattering (b) versus frequency $k r_{0}$ for bubbly gelatin.

Since the sample size is finite, the transmission is not completely diminished in the localization region, as expected [24]. In this particular case, there exists a narrow dip within the localization region between $k r_{0}=0.017$ and 0.024 , hereafter termed severe localization region, in which the most severe localization occurs. The waves are moderately localized between $k r_{0}=0.024$ and 0.077 , termed moderate localization region, due to fact that the finite size of sample still enables waves in this region to leak out [15]. We find from Fig. 2.3 that for such systems of internal resonances, the waves are not localized exactly at the internal resonance, rather at parameters slightly different from the resonance. This indicates that mere resonance does not promise localization, supporting the assertion of Rusek et al [33] and Alvarez et al [34]. 
To identify the phenomenon of localization by inspecting the correlation characteristics of the wave field in bubbly soft media, the total correlation functions are numerically studied for various frequencies and bubble parameters. Figure 2.4 illustrates the typical result of the comparison between the total correlation functions for bubbly gelatin at three particular frequencies chosen as below, within, and above the localization region: $k r_{0}=0.012,0.018$, and 0.1, referring to Fig. 2.3. Here the parameters of bubbles are identical with those used in Fig. 2.3. Observation of Fig. 2.4 clearly reveals that the total correlation decays rapidly along the distance $\xi$ in the case of $k r_{0}=0.018$, while the decrease of correlation with the increase of $\xi$ is very slow in the cases of $k r_{0}=0.012$ and $k r_{0}=0.1$. Such spatial correlation behaviors may be understood by considering the coherent and the diffusive portions of the transmission. Here the coherent portion is defined as $I_{C}=|\langle T\rangle|^{2}$, and the diffusive portion is $I_{D}=I-I_{C}$. Figure 2.5 plots the total transmission and the coherent portion versus frequency $k r_{0}$ for bubbly gelatin with the parameters used in Fig. 2.4. It is obvious that the coherent portion dominates the transmission for most frequencies, while the diffusive portion dominates within the localization region. This is in good agreement with the conclusion drawn by Ye et al for bubbly liquids (cf. see Fig. 1 in Ref. [24]). As a result, there exist strong correlations between pairs of field points even for a considerable large distance within the non-localized region where the wave propagation is predominantly coherent. Contrarily, within the localization region almost all the waves are trapped inside a spatial domain and the fluctuation of wave field at a spatial point fails in interacting effectively with any other point far from it. These results suggest that proper analysis of the spatial correlation behaviors may serve for a way that helps discern the phenomenon of localization in a unique manner.

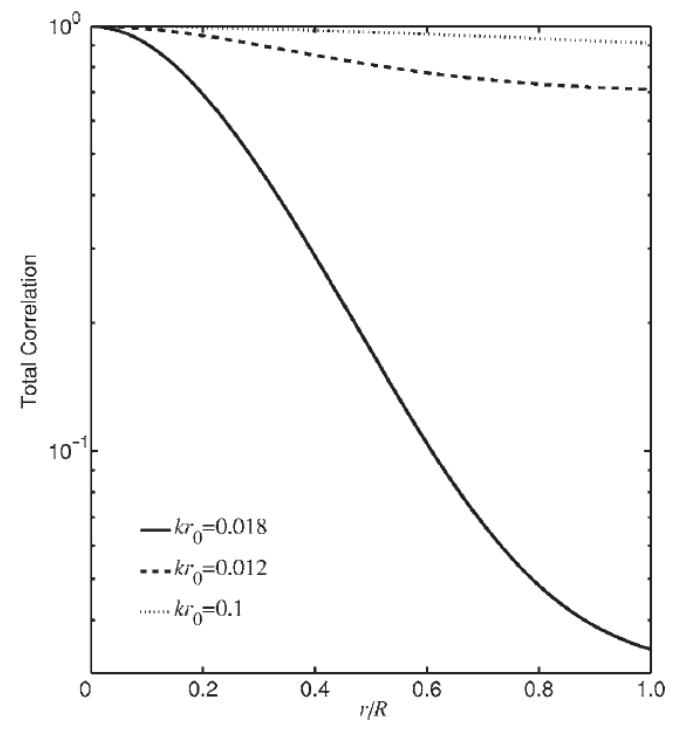

Fig. 2.4. The total correlation versus distance $\xi$ for bubbly gelatin at three particular frequencies chosen as below, within, and above the localization region, respectively. 


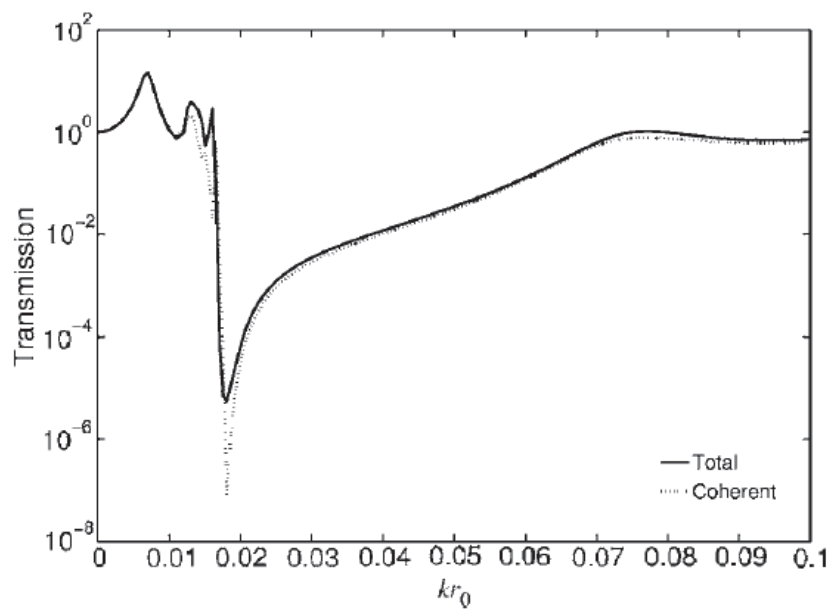

Fig. 2.5. The total transmission and the coherent portion versus frequency $k r_{0}$ for bubbly gelatin.

\section{Phase transition in acoustic localization in bubbly soft media}

In this section, we focus on the localization in bubbly soft medium with the effect of viscosity taken into account, by inspecting the oscillation phases of bubbles rather than the wave fields. It will be proved that the acoustic localization is in fact due to a collective oscillation of the bubbles known as a phenomenon of "phase transition", which helps to identify phenomenon of localization in the presence of viscosity.

\subsection{The influence of viscosity on acoustic localization}

So far, we have considered the localization property in a bubbly soft medium, which is regarded as totally elastic for excluding the effects of absorption that may lead to ambiguity in data interpretation. In practical situations, however, the existence of viscosity effect may notably affect the propagation of acoustic waves and then the localization characteristics in a bubbly soft medium. Note that the practical sample of a soft medium is in general assumed viscoelastic [6] and the existence of viscosity inevitably causes ambiguity in differentiating the localization effect from the acoustic absorption which might result in the spatial decrease of wave fields as well [36]. In the presence of viscoelasticity, the Lamé coefficients of the soft medium may be rewritten as below:

$$
\lambda=\lambda_{e}+\lambda_{v} \frac{\partial}{\partial t}, \mu=\mu_{e}+\mu_{v} \frac{\partial}{\partial t},
$$

where $\lambda_{e}$ and $\mu_{e}$ are the elastic Lamé coefficients, $\lambda_{v}$ and $\mu_{v}$ are viscosity factors given by Kelvin-Voigt viscoelastic model. In the following we shall assume $\lambda_{v}=0$, as is usually done for a soft medium [35]. The viscosity factor $\mu_{v}$ may be manually adjusted in the numerical simulations to inspect the sensibility of the results to the absorption effects. 
Note that the acoustic wave is a simple harmonic wave of angular frequency $\omega$. Then the longitudinal wave number in the soft viscoelastic medium becomes a complex number as $\tilde{k}=k^{\prime}+i k^{\prime \prime}=\omega / \tilde{c}_{l}$. Here the real and the imaginary parts represent the propagation and the attenuation of the longitudinal wave in a soft viscoelastic medium, respectively, and $\tilde{c}_{l}$ refers to the effective speed of the wave. For the acoustic wave that propagates in a soft viscoelastic medium permeated with bubbles, the influence of the viscosity effect may be ascribed to two aspects: (1) the propagation of the acoustic wave in a soft viscoelastic medium should be described by a series of complex parameters instead of the corresponding real parameters (i.e., $k \rightarrow \tilde{k}, c_{l} \rightarrow \tilde{c}_{l}$, etc.) to account for the absorption effects; (2) the dynamical behavior of an individual bubble will be greatly affected by the friction damping of pulsation that results from the viscoelastic solid wall. The incorporation of the effect of acoustic absorption due to viscosity effects amounts to adding a term $v \cdot d U / d t$ in the dynamical equation of a single bubble in a soft elastic medium [8]. Here $v=4 \mu_{v} /\left(\rho r_{0}^{2}\right)$ is a coefficient characterizing the effect of acoustic absorption. By seeking the linear solution of the modified dynamical equation in a same manner as in Section 2.3, one may derive the scattering function $f$ of a single bubble in a soft viscoelastic medium, as follows:

$$
f=\frac{r_{0}}{\left(\omega_{0}^{2} / \omega^{2}-1-i \omega r_{0} / c_{l}-i \nu / \omega\right)},
$$

where $\omega_{0}$ refers to the resonance frequency of an individual bubble in a soft medium. On condition that the soft medium is totally elastic, the expression of the scatter function $f$ degenerates to Eq. (2.6) due to the vanishing of the term $-i v / \omega$. In such a case, the acoustic field in any spatial point can thus be solved exactly in a same manner as in Section 2.4.

By rewriting the complex coefficient $A_{i}$ in Eq. (2.10) as $A_{i}=\left|A_{i}\right| \exp \left(i \theta_{i}\right)$ with the modulus and the phase physically represent the strength of secondary source and the oscillation phase, respectively. For the $i$ th bubble, it is convenient to assign a two-dimensional unit phase vector, $\boldsymbol{u}_{i}=\cos \theta_{i} \hat{x}+\sin \theta_{i} \hat{y}$ to the oscillation phase of the bubble with $\hat{x}$ and $\hat{y}$ being the unit vectors in the $x$ and $y$ directions, respectively. The phase of emitting source is set to be zero. Thereby the oscillation phase of every bubble is mapped to a two-dimensional plane via the introduction of the phase vectors and may be easily observed in the numerical simulations by plotting the phase vectors in a phase diagram.

In actual experiments, it is the variability of signal that is often easier to analysis [36]. Hence the behavior of the phases of the oscillating bubbles may be readily studied by inspecting the fluctuation of the oscillation phase of bubbles is investigated as well. Here the fluctuation of the phase of bubbles is defined as follows [36]:

$$
\delta \theta=\left[\frac{1}{N} \sum_{\mathrm{i}=1}^{N} \theta_{\mathrm{i}}^{2}-\bar{\theta}^{2}\right]^{2},
$$

where $\bar{\theta}=\frac{1}{N} \sum_{\mathrm{i}=1}^{N} \theta_{\mathrm{i}}$ is the averaged phase. 


\subsection{Localization and phase transition in bubbly soft media}

Figure 3.1 displays the typical results of the phase diagrams for a bubbly gelatin at different driving frequencies, with the values of viscosity factors manually adjusted to study the influence of the effect of acoustic absorption. Three particular frequencies are employed (See Fig. 2.3): $\omega r_{0} / c_{1}=0.01$ (Fig. 3.1(a), below the localization region), $\omega r_{0} / c_{1}=0.1$ (Fig. 3.1(b), above the localization region), and $\omega r_{0} / c_{1}=0.02$ (Figs. 3.1(c) and (d), within the localization region). In a phase diagram, each circle and the corresponding arrow refer to the three-dimensional position and the phase vector of an individual bubble, respectively. In Figs. 3.1(a-c) we choose the viscosity factor as $\mu_{\mathrm{v}}=0$, i.e., the soft medium that serves as the host medium is assumed totally elastic; while in Fig. 3.1(d) the value of viscosity factor is set to be $\mu_{\mathrm{v}}=50 \mathrm{P}$ $(1 \mathrm{P}=0.1 \mathrm{~Pa} \cdot \mathrm{s})$. For a comparison we also examine the spatial distribution of the wave fields and plot the transmissions as a function of the distance from the source in Fig. 3. 2 in cases corresponding to Fig. 3.1. Note that the energy flow of an acoustic wave is conventionally $\boldsymbol{J} \sim|p|^{2} \nabla \theta_{\mathrm{i}}$. This mathematical relationship reveals the fact that the gradient of oscillation phases of bubbles is crucial for the occurrence of localization. Apparently, when the oscillation phases of different bubbles exhibit a coherent behavior (i.e. $\theta_{\mathrm{i}}$ is a constant) while $|p|$ is nonzero, the acoustic energy flow will stop and the acoustic wave will thereby be localized within a spatial domain [36]. Moreover, such coherence in oscillation phases of bubbles is a unique feature of the phenomenon of localization that results from the multiple scattering of waves, but lacks when other mechanism such as absorption effect dominates, as will be discussed later. Consequently, it should be promising to effectively identify the localization phenomenon by giving analysis to the oscillation phases of bubbles and seeking their ordering behaviors.

It is apparent in Figs. 3.1(a) and (b) that the phase vectors pertinent to different bubbles point to various directions as the driving frequency of the source lies outside the localization region. In other words, the oscillation phases of the bubbles located at different positions in a bubbly soft medium are random in non-localized states. Correspondingly, the curves 1 (thin solid line) and 2 (thin dashed line) in Fig. 3.2 shows that the non-localized waves remain extended and can propagate through the bubble cloud. As observed in Fig. 3.1(c), however, the phase vectors located at different spatial positions point to the same direction when localization occurs, which indicates that the oscillation phases of all bubbles remain constant and the energy flow of the wave stops. The transition from the non-localized state to the localized state of the wave can be interpreted as a kind of "phase transition", which is characterized by the unusual phenomenon that all the bubbles pulsate collectively to efficiently prohibit the acoustic wave from propagating [10]. Such a concept of phase transition is physically consistent with the order-disorder phase transition in a ferromagnet [37]. Note that the phase of emitting source is assumed to be zero in the numerical simulations, i.e., the phase vector at the source points to positive $\hat{x}$ direction, while all the phase vectors in Fig. 3.1(c) point to the negative $\hat{x}$-axis. This means that as the localization occurs, almost all bubbles tend to oscillate completely in phase but exactly out of phase with the source, which leads to the fact that the localized acoustic energies are trapped within a small spatial domain adjacent to the source as shown by the curve 3 (thick solid line) in Fig. 3.2. These numerical results are consistent with the previous conclusions obtained for bubbly water and bubbly soft elastic media $[10,36]$. Therefore it is reasonable to conclude that such a phenomenon of phase transition is the intrinsic physical mechanism from which the acoustic localization stems. 

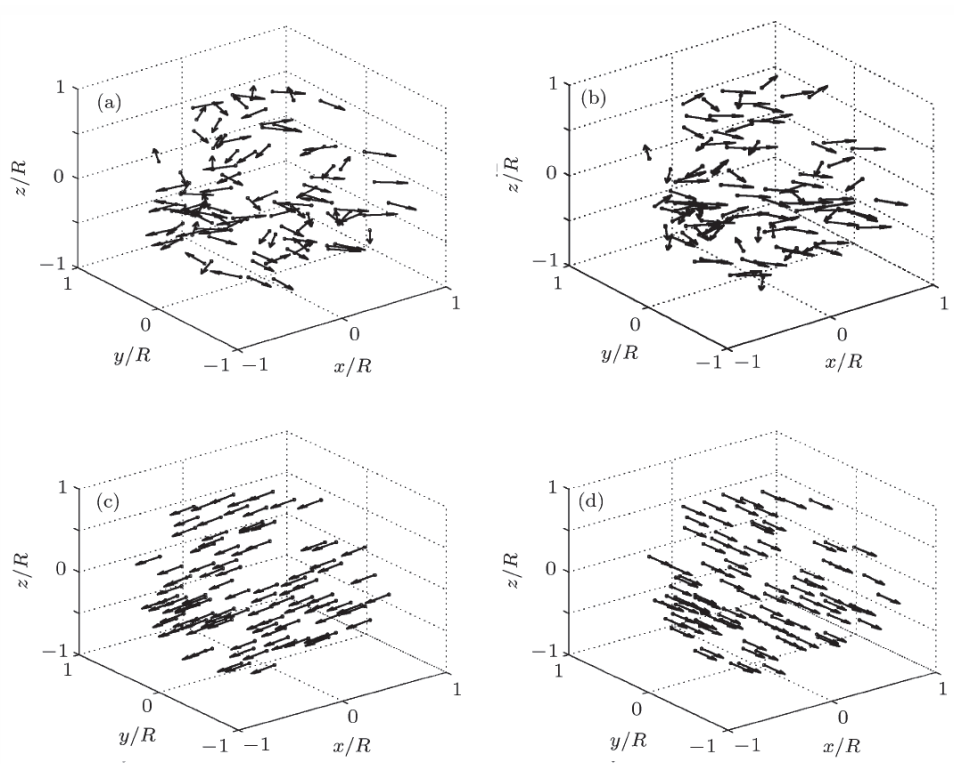

Fig. 3.1. The phase diagrams for the oscillating bubbles in a bubbly gelatin with different structural parameters: (a) $\omega r_{0} / c_{1}=0.01, \mu_{\mathrm{v}}=0$; (b) $\omega r_{0} / c_{1}=0.1, \mu_{\mathrm{v}}=0$; (c) $\omega r_{0} / c_{1}=0.02, \mu_{\mathrm{v}}=0$; (d) $\omega r_{0} / c_{1}=0.02, \mu_{\mathrm{v}}=50 \mathrm{P}$.

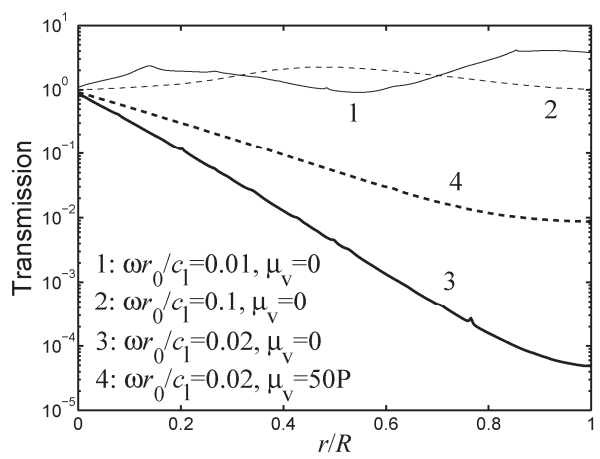

Fig. 3.2. Transmissions versus the distance from the source in a bubbly gelatin with different structural parameters.

Note that the effect of acoustic absorption has been completely excluded in Figs. 3.1(a-c) which may cause ambiguity in identifying the phenomenon of localization. It is thus of much more practical significance to investigate the localization properties in the case where soft medium is assumed viscoelastic, and the corresponding results are shown in the phase diagram given by Fig. 3.1 (d) as well as the comparison between transmissions versus $r$ in Fig. 3.2. As the viscosity factors of the soft medium are manually increased, the phenomena of phase transition can be identified in a bubbly soft viscoelastic medium provided that the 
driving frequency of acoustic wave falls within the localization region. Meanwhile, exponential decay of the wave fields with respect to the distance from the source is shown by the curve 4 (thick dashed line) in Fig. 3.2. Observation of Fig. 3.1(d) and Fig. 3.2 apparently manifests that, however, the adjustment of the values of the viscosity factors leads to changes of the direction to which all the phase vectors point collectively varies and the decay rates of the transmissions versus $r$.

For a bubbly soft viscoelastic medium, it is still possible to achieve the acoustic localization since the condition can be satisfied that the oscillation phases of bubbles at any spatial points remain constant, but the extents of localization are necessarily affected by the presence of viscosity effect. It is thus difficult to differentiate the phenomenon of acoustic localization from that of the acoustic absorption without referring to the analysis of the behavior of the phases of bubbles [11]. Notice that in Fig. 3.1, as the viscosity factors are gradually enhanced, the angles between the directions of the phase vectors and the negative $x$-axis increase. This means that the phase-opposition states between the oscillations of all the bubbles and the source as well as the extents to which the acoustic wave is localized are weaken due to the enhancement of the viscosity. Therefore it may be inferred that the occurrence of phase transition in a bubble soft medium is a criterion for identifying the phenomenon of localization, while the localization extents can be predicted by accurately analyzing the relationship between the oscillation phases of the bubbles and the source.

It is convenient to employ a phase diagram method for observing the collective phase properties of the bubbles and thereby seeking the existence of the phenomenon of phase transition, however the values of the oscillation phase of each bubble could not be directly read via the phase diagrams in a precise manner. We then illustrate the statistical properties of the parameters of $\langle\theta\rangle$ for all the bubbles in Fig. 3.3 for a more explicit observation of the values of oscillation phases of the bubbles. Here $\langle\cdot\rangle$ denotes the ensemble average over random configurations of bubble clouds, $p_{\theta}(\theta)$ is defined as the probability that the values of $\langle\theta\rangle$ fall between $\theta$ and $\theta+\Delta \theta$, i.e., $\theta \leq\langle\theta\rangle\langle\theta+\Delta \theta$, with $\Delta \theta$ referring to the difference between the two neighbor discrete values of $\theta$. And the values of $p_{\theta}(\theta)$ have been normalized such that the total probability equals 1. In Fig. 3.3 three particular values of viscosity factors are considered: $\mu_{\mathrm{v}}=0$ (curve 3, thick solid line), 50P (curve 4, thick dashed line), 200P (curve 5, thick dotted line). It is obvious in Fig. 3.3 that: (1) Outside the localization region, as shown by the thin curves 1 (solid line) and 2 (dashed line), the values of oscillation phases $\langle\theta\rangle$ exhibit large extents of randomnesses, which indicates a lack of the above-mentioned collective behavior of the bubble oscillation crucial for the existence of localization, in accordance with the results shown in Figs. 3.1(a) and (b). (2) When the phenomenon of localization occurs, the oscillation phases almost remain constant for bubbles located at different spatial points, which is illustrated by the delta-function shapes of the thick curves 3-5. It is also noteworthy that the oscillation phase of each bubble approximates $-\pi$ in an elastic medium, and that the presence of the viscosity effect does not change such a phenomenon of phase transition but leads to a larger average value of oscillation phases $\langle\theta\rangle$. A monotonic increase of the values of the oscillation phases of bubbles is clearly observed as the viscosity factors are gradually enhanced. In the soft medium with viscosity factor $\mu_{\mathrm{v}}=50 \mathrm{P}$, the values of $\langle\theta\rangle$ nearly equal $-0.45 \pi$ for all the bubbles, and $\langle\theta\rangle$ approximate $-0.15 \pi$ for the case of $\mu_{\mathrm{v}}=200 \mathrm{P}$. 


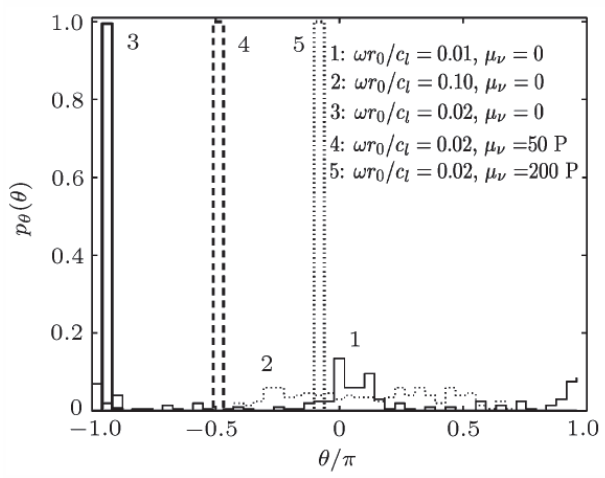

Fig. 3.3. The comparison between the statistical behaviors of the oscillation phases of bubbles in a bubbly gelatin with different structural parameters.

The principal influence of the viscosity effect on the localization property in a bubbly soft medium attributes intrinsically to two aspects of physical mechanism. The localization phenomenon in inhomogeneities had been extensively proved to stem from the important multiple scattering processes between scatterers. In a viscoelastic medium the recursive process of multiple scattering could not be well established due to the effect of acoustic absorption caused by the viscosity, which necessarily impairs the extent to which the acoustic wave can be localized. For an individual bubble pulsating in a viscoelastic medium, on the other hand, the oscillation will be hindered by the friction damping caused by the viscoelastic solid wall. While the bubble in an elastic soft medium can behave like a high quality factor oscillator [2], the increase of viscosity factors will definitely reduce the quality factor that is defined as $Q=\omega / v$ and then the strength of the resonance response of bubble to the incident wave. This prevents the bubbles from becoming effective acoustic scatterers, which is crucial for the localization to take place [24]. As a result, it is perceivable that the increase of the viscosity effects diminishes the extent to which all bubbles pulsate out of phase with the source, and a complete prohibition of acoustic wave could not be attained.

Figure 3.4 displays the fluctuations of the oscillation phases of bubbles $\delta \theta$ as a function of the normalized frequency $\omega r_{0} / c_{1}$ in a bubbly gelatin for four particular values of viscosity factors: $\mu_{\mathrm{v}}=0,5 \mathrm{P}, 50 \mathrm{P}$ and 500P. Note also that the fluctuations of the phases approaches zero at the zero frequency limit due to the negligibility of the scattering effect of bubbles. The phenomena of phase transitions can be clearly observed characterized by significant reductions of the fluctuations within particular ranges of frequencies whose locations are in good agreement with the corresponding frequency regions where the localization occurs. This is consistent with the previous results obtained for bubbly water. Moreover, it is apparently seen that the amounts to which the fluctuations $\delta \theta$ decrease can act as reflections of the extents of the acoustic localizations. In a bubbly viscoelastic soft medium, such a phenomenon of phase transition persists within the localization region, while the increase of the value of viscosity factor leads to a weaker reduction of the fluctuation of phases. In the particular case where the viscosity effects are extremely strong, i.e., $\mu_{\mathrm{v}}=500 \mathrm{P}$, the localization is absent due to the fact that the effects of multiple scattering and the bubble resonance are severely destroyed, and the phenomenon of phase transition could not be 
identified. The comparison of Figs. 3.1-3.4 proved that the phenomenon of phase transition is a valid criterion of the existence of acoustic localization in such a medium, and the values of the oscillation phases of the bubbles help to determine the extent to which the acoustic waves are localized. Consequently it is fair to conclude that the proper analysis of the oscillation phases of bubbles can indeed act as an efficient approach to identify the phenomenon of acoustic localization in the practical samples of bubbly soft media for which the viscosity effects are generally nontrivial. The important phenomenon of phase transition is an effective criterion to determine the existence of localization, while the extent to which the acoustic wave is localized may be estimated by inspecting the values of the oscillation phases or the reduction amount of the phase fluctuation.

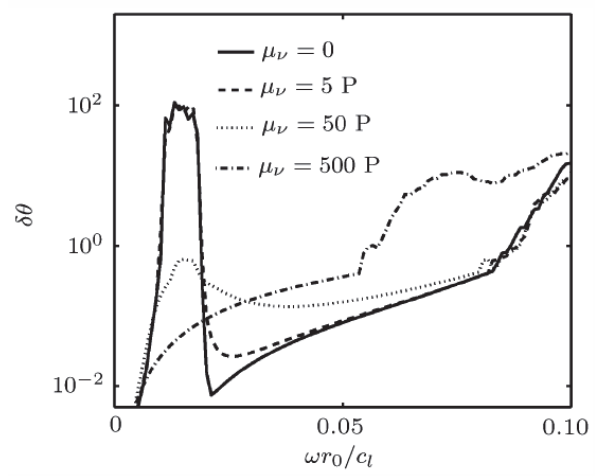

Fig. 3.4. The comparison between the fluctuations of the oscillation phases of bubbles versus frequency in a bubbly gelatin with different values of viscosity factors.

\section{Effective medium method for sound propagation in bubbly soft media}

In this section, we discuss the nonlinear acoustic property of soft media containing air bubbles and develop an EMM to describe the strong acoustic nonlinearity of such media with the effects of weak compressibility, viscosity, surrounding pressure, surface tension, and encapsulating shells incorporated. The advantages as well as limitations of the EMM are also briefly discussed.

\subsection{Bubble dynamics}

Consider an encapsulated gas bubble surrounded by a soft viscoelastic medium. When in equilibrium, the gas pressure in the bubble is denoted $P_{g}$, and the pressure infinitely far away is $P_{\infty}$. For the case where the equilibrium pressure equals the surrounding pressure (i.e. $P_{g}=P_{\infty}$ ), the shear stress is uniform throughout the soft medium. Such a case is referred to as an initially unstressed state, for which the equilibrium values of the inner and outer radius of the bubble are designated $R_{0}$ and $R_{s 0}$, respectively. In the general case, however, the encapsulated bubble may be pressurized, such that $P_{g} \neq P_{\infty}$. Such a case is denoted as a prestressed case due to the fact that a nonuniform shear stress is generated inside the medium to balance the pressure difference. For a prestressed cases we define the 
equilibrium values of the inner and outer radius as $R_{1}$ and $R_{s 1}$, respectively. The geometry is shown in Fig. 4.1. Figure 4.1(a) shows an unstressed case where one has $R_{0}=R_{1}$ and $R_{s 0}=R_{s 1}$. In the cases where $P_{g}<P_{\infty}$, however, it is apparently that the pressure difference between $P_{g}$ and $P_{\infty}$ will force the bubble to shrink, and one thus has $R_{0}>R_{1}$ and $R_{s 0}>R_{s 1}$, as illustrated in Fig. 4.1(b). In contrast, one has $R_{0}<R_{1}$ and $R_{s 0}<R_{s 1}$ if $P_{g}>P_{\infty}$. As the bubble oscillates, the instantaneous values of the inner and outer radius are defined as $R(t)$ and $R_{s}(t)$, respectively.
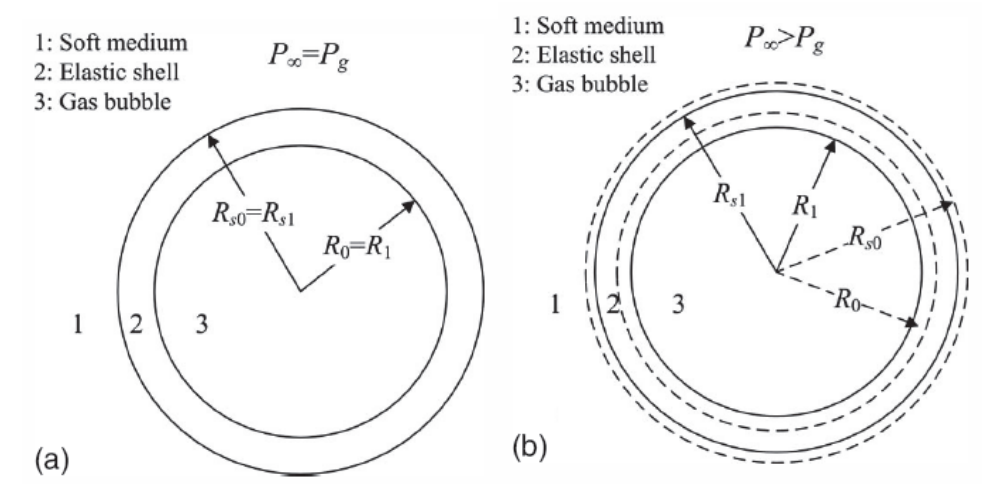

Fig. 4.1. Geometry of an encapsulated gas bubble in a soft medium in (a) an initially unstressed state and (b) a prestressed state.

Zabolotskaya et al [6] has studied the nonlinear dynamics in the form of a Rayleigh-Plessetlike equation for an individual bubble in such a model, and provided the approaches to include the effects of compressibility, surface tension, viscosity, and an encapsulating shell. Note that Eq. (53) in Ref. [6] accounts for the effects of surface tension, viscosity, and shell but applies only to the case of an incompressible medium. Adding the compressibility term $\left(d^{3} w / d t^{3}\right) / c_{m}$ that accounts for the radiation damping to the left hand side of this equation, one readily obtain the equation that describes the nonlinear oscillation of a single bubble in a soft medium, as follows:

$$
\begin{aligned}
& F(R) R \frac{d^{2} R}{d t^{2}}+\frac{3}{2} G(R)\left(\frac{d R}{d t}\right)^{2}-\frac{1}{c_{m}} \frac{d^{3} w}{d t^{3}}=\frac{1}{\rho_{m}}\left\{P_{g}\left(\frac{R_{1}}{R}\right)^{3 \gamma}-P_{\infty}\right. \\
&\left.-P_{e}(R)-\frac{2 \sigma_{g}}{R}-\frac{2 \sigma_{m}}{R_{s}}-\frac{4}{R} \frac{d R}{d t}\left[\eta_{s}\left(1-\frac{R^{3}}{R_{s}^{3}}\right)+\eta_{m} \frac{R^{3}}{R_{s}^{3}}\right]\right\},
\end{aligned}
$$

In the preceding expression, the parameter $w$ is defined as $w=R^{3} / 3$, $P_{\sigma}=2 \sigma_{g} / R+2 \sigma_{m} / R_{s}$ is the effective pressure due to the surface tension with $\sigma_{g}$ and $\sigma_{m}$ being the surface tensions at the inner gas-shell interface and the outer shell-medium interface, respectively, and $\psi$ is the dissipation function that is found to be $\psi=8 \pi R(d R / d t)^{2}\left[\eta_{s}\left(1-R^{3} / R_{s}^{3}\right)+\eta_{m} R^{3} / R_{s}^{3}\right]$ with $\eta_{s}$ and $\eta_{m}$ being the shear viscosity coefficients in the shell region and in the medium, respectively, the parameters of $F(R)$ and $G(R)$ are given as 


$$
F(R)=\frac{\rho_{s}}{\rho_{m}}+\left(1-\frac{\rho_{s}}{\rho_{m}}\right) \frac{R}{R_{s}}, G(R)=\frac{\rho_{s}}{\rho_{m}}+\left(1-\frac{\rho_{s}}{\rho_{m}}\right)\left(\frac{4}{3}-\frac{1}{3} \frac{R^{3}}{R_{s}^{3}}\right) \frac{R}{R_{s}}
$$

with $\rho_{s}$ and $\rho_{m}$ are the mass densities of the shell and the surrounding medium, respectively, and $P_{e}(R)$ refers to the effective pressure due to the strain energy stored in shear deformation of both the shell and the medium, defined as [6]

$$
P_{e}(R)=4 \int_{r}^{R_{s}}\left(\frac{\partial \varepsilon_{s}}{\partial I_{1}}+\frac{r^{2}}{\tilde{r}^{2}} \frac{\partial \varepsilon_{s}}{\partial I_{2}}\right)\left(\frac{r^{2}}{\tilde{r}^{2}}-\frac{\tilde{r}^{4}}{r^{4}}\right) \frac{d r}{r}+4 \int_{R_{s}}^{\infty}\left(\frac{\partial \varepsilon_{m}}{\partial I_{1}}+\frac{r^{2}}{\tilde{r}^{2}} \frac{\partial \varepsilon_{m}}{\partial I_{2}}\right)\left(\frac{r^{2}}{\tilde{r}^{2}}-\frac{\tilde{r}^{4}}{r^{4}}\right) \frac{d r}{r},
$$

where $\varepsilon=\varepsilon\left(I_{1}, I_{2}, I_{3}\right)$ refers to the strain energy density with $I_{1}, I_{2}, I_{3}$ being the principal invariants of Green's deformation tensor, $r$ and $\tilde{r}$ refer to the Eulerian and the Lagrangian coordinates, respectively, the subscripts $s$ and $m$ refer to the shell and for the surrounding medium, respectively.

For the convenience of the following investigation, we will evaluate Eq. (4.1) here in the quadratic approximation by rewriting it into another form for the perturbation in bubble volume defined as $U=4 \pi\left(R^{3}-R_{1}^{3}\right) / 3$. For a soft medium Mooney's constitutive relation [38] is the most widely used model equation and has been adopted by many previous studies regarding the nonlinear dynamics of a bubble in such a medium [3-6]. For facilitating the comparison with the previous studies, therefore, we employ Mooney's relation to evaluate the effective pressure $P_{e}(R)$, as follows:

$$
\varepsilon_{p}=\mu_{p}\left[(1+\chi)\left(I_{1}-3\right)+(1-\chi)\left(I_{2}-3\right)\right] / 4, \quad p=s, m
$$

where $\mu_{p}$ is the shear modulus.

Substituting Eq. (4.3) into Eq. (4.2) and expanding $P_{e}(R)$ to quadratic order, one may derive an analytical approximation of $P_{e}(R)$, as follows:

$$
P_{e}(R)=P_{e}(\zeta)=P_{e}\left(\zeta_{1}\right)+\left(\zeta-\zeta_{1}\right) P_{e}^{\prime}\left(\zeta_{1}\right)+\left(\zeta-\zeta_{1}\right)^{2} P_{e}^{\prime \prime}\left(\zeta_{1}\right) / 2,
$$

where $\zeta=R / R_{0}, \zeta_{1}=R_{1} / R_{0}$, the primes represent derivatives with respect to $\zeta$, and the parameters of $P_{e}(\zeta), P_{e}^{\prime}(\zeta)$, and $P_{e}^{\prime \prime}(\zeta)$ are given as below:

$$
\begin{gathered}
P_{e}(\zeta)=\mu_{m} \int_{0}^{a}\left[(1+\chi)\left(y x^{-1}-y^{7} x^{-8}\right)+(1-\chi)\left(y^{-1}-y^{5} x^{-6}\right)\right] d x \\
+\mu_{s} \int_{a}^{1}\left[(1+\chi)\left(y x^{-1}-y^{7} x^{-8}\right)+(1-\chi)\left(y^{-1}-y^{5} x^{-6}\right)\right] d x, \\
P_{e}^{\prime}(\zeta)=\mu_{s} \zeta^{2} \int_{0}^{a}\left[(1+\chi)\left(7 y^{10} x^{-8}-y^{4} x^{-2}\right)+(1-\chi)\left(y^{2}+5 y^{8} x^{-6}\right)\right] d x \\
+\mu_{m} \zeta^{2} \int_{a}^{1}\left[(1+\chi)\left(7 y^{10} x^{-8}-y^{4} x^{-2}\right)+(1-\chi)\left(y^{2}+5 y^{8} x^{-6}\right)\right] d x, \\
P_{e}^{\prime \prime}(\zeta)=\mu_{s} \zeta^{4} \int_{0}^{a}\left[(1+\chi)\left(4 y^{7} x^{-2}-70 y^{13} x^{-8}\right)-(1-\chi)\left(2 y^{5}+40 y^{11} x^{-6}\right)\right] d x \\
+\mu_{m} \zeta^{4} \int_{a}^{1}\left[(1+\chi)\left(4 y^{7} x^{-2}-70 y^{13} x^{-8}\right)-(1-\chi)\left(2 y^{5}+40 y^{11} x^{-6}\right)\right] d x \\
+2 P_{e}^{\prime}(\zeta) / \zeta,
\end{gathered}
$$


where $x=R_{0} / \tilde{r}, y=R_{0} / r, a=R_{0} / R_{s 0}$.

Substituting Eq. (4.4) into Eq. (4.1), one obtains the expansion of Eq. (4.1) to quadratic order in $U$, as follows:

$$
\begin{gathered}
\frac{d^{2} U}{d t^{2}}+\delta_{1} \frac{d U}{d t}+\omega_{1}^{2} U-\frac{R_{1}}{F_{1} c_{m}} \frac{d^{3} U}{d t^{3}}=G_{1} U^{2}+\delta_{2} U \frac{d U}{d t} \\
+H_{1}\left[\left(\frac{d U}{d t}\right)^{2}+2 U \frac{d^{2} U}{d t^{2}}\right]-e_{1} P_{A^{\prime}}
\end{gathered}
$$

where $P_{A}(t)=P_{\infty}-P_{0}$ is the applied acoustic pressure with $P_{0}$ being the pressure at infinity in the absence of sound, $\omega_{1}=\sqrt{\omega_{g}^{2}+\omega_{e}^{2}-\omega_{\sigma}^{2}}$ is the nature frequency of bubble for which the components are given as

$$
\omega_{g}^{2}=3 \gamma P_{g} D_{1}, \omega_{e}^{2}=P_{e}^{\prime}\left(\zeta_{1}\right) \zeta_{1} D_{1}, \omega_{\sigma}^{2}=2 D_{1} R_{1}^{-1}\left(\sigma_{g}+\sigma_{m} \gamma_{R}^{4}\right),
$$

$\gamma$ is the ratio of specific heats which is chosen as $\gamma=1.4$ since the we only consider air bubbles in the present study, $\delta_{1}$ and $\delta_{2}$ are the viscous damping coefficients at linear and quadratic order, respectively, defined as

$$
\delta_{1}=4 D_{1}\left[\eta_{s}\left(1-\gamma_{R}^{3}\right)+\eta_{m} \gamma_{R}^{3}\right], \delta_{2}=24 q_{1} D_{1}\left[\eta_{s}\left(1-\gamma_{R}^{6}\right)+\eta_{m} \gamma_{R}^{6}\right],
$$

$G_{1}$ is the nonlinearity coefficient associated with gas compressibility, elasticity, and surface tension that is defined as

$$
G_{1}=q_{1}\left[3(\gamma+1) \omega_{g}^{2}+\left(2-\frac{P_{e}^{\prime \prime}\left(\zeta_{1}\right) \zeta_{1}}{P_{e}^{\prime}\left(\zeta_{1}\right)}\right) \omega_{e}^{2}-4 \omega_{\sigma}^{2}+\frac{8 \sigma_{m} R_{1}}{\rho_{m} F_{1} R_{s 1}^{4}}\left(1-\gamma_{R}^{3}\right)\right],
$$

and the parameters of $F_{1}, H_{1}, e_{1}, q_{1}, \gamma_{\rho}, \gamma_{R}$ and $D_{1}$ are given as follows

$$
\begin{aligned}
F_{1} & =\gamma_{\rho}+\left(1-\gamma_{\rho}\right) \gamma_{R}, H_{1}=q_{1} F_{1}^{-1}\left[\gamma_{\rho}+\left(1-\gamma_{\rho}\right) \gamma_{\rho}^{4}\right], e_{1}=4 \pi D_{1} R_{1}^{3}, q_{1}=1 /\left(8 \pi R_{1}^{3}\right), \gamma_{\rho}=\rho_{s} / \rho_{m}, \\
\gamma_{R} & =R_{1} / R_{s 1}, D_{1}=1 /\left(\rho_{m} F_{1} R_{1}^{2}\right) .
\end{aligned}
$$

\subsection{Effective medium method}

\subsubsection{Effective medium}

We now study the propagation of a plane acoustic wave in an infinite soft medium containing random encapsulated bubbles, subject to the condition that the volume content of the bubbles is small but the number of bubbles on a scale of wavelength order is large. Then it can be proved that the multiple scattering effects are negligible [39] and the homogeneous approximation well known for liquid containing bubbles can be employed [3]. Consider a small volume element of the medium of length $d x_{i}$ in the $x_{i}$ direction in the Cartesian coordinate $(i=1,2,3)$ that is sufficiently large to include a number of bubbles. In the present study, we shall focus our attention on the cases where the amplitude of wave is small, for the purpose of investigating the strong physical nonlinearity of such a class of media [3,4]. Then the dynamic nonlinearity is negligible that dominates only on condition that the amplitude of wave is finite. According to the stress-strain relationship and 
neglecting the contribution of the gas inside the bubbles, the stress tensor may be expressed as (see Ref. [7], pp. 10)

$$
\sigma_{i k}^{s}=(1-\beta)\left[K u_{\| l}^{s} \delta_{i k}+2 \mu\left(u_{i k}^{s}-\delta_{i k} u_{l l}^{s} / 3\right)\right],
$$

where $\sigma_{i k}^{s}$ and $u_{i k}^{s}$ are the stress tensor and the strain tensor of the solid phase, $\beta$ is the total volume fraction of the bubbles, $K=\lambda+2 \mu / 3$ is the bulk modulus, $\delta_{i k}$ refers to the Kronecker delta which is defined as

$$
\delta_{i k}=\left\{\begin{array}{ll}
1, & i=k \\
0, & i \neq k
\end{array} .\right.
$$

On the other hand, the volume element of the bubbly soft medium may be regarded as a volume element of "effective" medium that is homogeneous and is described by effective acoustical parameters. The stress tensor of the effective medium may be given as below:

$$
\tilde{\sigma}_{i k}=\tilde{K} \tilde{u}_{l l} \delta_{i k}+2 \tilde{\mu}\left(\tilde{u}_{i k}-\delta_{i k} \tilde{u}_{l l} / 3\right),
$$

where $\tilde{\sigma}_{i k}$ and $\tilde{u}_{i k}$ are the stress tensor and the strain tensor of the effective medium, respectively, $\tilde{K}=\tilde{\lambda}+2 \tilde{\mu} / 3$ is the effective bulk modulus with $\tilde{\lambda}$ and $\tilde{\mu}$ being the effective Lamé coefficients of the effective medium.

\subsubsection{Influence of bubble oscillation}

As the acoustic wave propagates in the bubbly medium, the volume of the bubbles will change due to the oscillation of the bubbles driven by the acoustic wave. As a result, the variation of the volume element includes the compression of the elastic phase and the variation of the total volume of the bubbles. Then one has

$$
\tilde{\theta}=\theta^{s}(1-\beta)+V_{t},
$$

where $\tilde{\theta}=\tilde{u}_{11}+\tilde{u}_{22}+\tilde{u}_{33}$ and $\theta^{s}=u_{11}^{s}+u_{22}^{s}+u_{33}^{s}$ are the volume changes of the effective medium and the elastic phase, respectively, $V_{t}$ is the variation of the specific volume of bubbles.

As the bubble distorts under the action of the shear deformation, the principal radii of curvature of the surface will change. This will certainly change the effect of surface tension and then the bubble volume. In the present study, however, the bubbles are assumed spherical, and such an effect is then negligible that does not change the nature of the bubble dynamics. Then it is fair to assume approximately that the pure shear deformation of the volume element will not affected by the existence of bubbles. Then one has

$$
\sigma_{i k}^{s}=\tilde{\sigma}_{i k}, u_{i k}^{s}=\tilde{u}_{i k} . \quad i \neq k
$$

Substituting Eq. (4.9) into Eqs. (4.6) and (4.7) yields $\tilde{\mu}=\mu(1-\beta)$.

From Eqs. (4.6) and (4.7) one readily obtains

$$
\sigma_{11}^{s}+\sigma_{22}^{s}+\sigma_{33}^{s}=3(1-\beta)(\lambda+2 \mu / 3) \theta^{s}
$$




$$
\tilde{\sigma}_{11}+\tilde{\sigma}_{22}+\tilde{\sigma}_{33}=3(\tilde{\lambda}+2 \tilde{\mu} / 3) \tilde{\theta}
$$

Under the action of an applied force, the element of effective medium is defined to produce the same stress as the element of the bubbly medium. Hence one has

$$
\sigma_{11}^{s}+\sigma_{22}^{s}+\sigma_{33}^{s}=\tilde{\sigma}_{11}+\tilde{\sigma}_{22}+\tilde{\sigma}_{33}
$$

Substituting Eqs. (4.8-4.10) into Eqs. (4.6) and (4.7) yields (for $\lambda>>\mu$ )

$$
\tilde{C}_{11} \theta^{s}=C_{11}\left(\theta^{s}-V_{t}\right)
$$

where $C_{11}=\lambda+2 \mu, \tilde{C}_{11}=\tilde{\lambda}+2 \tilde{\mu}$ are the elastic modulus of the soft medium and the effective medium, respectively.

For the purpose of solving the unknown quantity $V_{t}$, it is necessary to obtain the variation of the volume of an individual bubble $U$ which is described by the equation for the oscillation of a bubble given by Eq. (4.5). In Eq. (4.5), the acoustic pressure $P_{A}$ accounts for the driving force of the oscillation of the bubble. Due to the fact the shear wave does not change the volume of the bubble, the driving force of the bubble oscillation is not affected by the shear wave but determined by the total radial force exerted by the incident wave [3]. According to Eq. (17) in Ref. [3] one has

$$
P_{A}=\frac{\lambda+2 \mu / 3}{\lambda+2 \mu} \sigma \simeq \sigma
$$

where $\sigma=\tilde{\sigma}_{11}$ is the pressure generated by the incident wave.

Substituting Eq. (4.13) into Eq. (4.5) yields

$$
\begin{gathered}
\frac{d^{2} U}{d t^{2}}+\delta_{1} \frac{d U}{d t}+\omega_{1}^{2} U-\frac{R_{1}}{F_{1} c_{m}} \frac{d^{3} U}{d t^{3}}=G_{1} U^{2}+\delta_{2} U \frac{d U}{d t} \\
+H_{1}\left[\left(\frac{d U}{d t}\right)^{2}+2 U \frac{d^{2} U}{d t^{2}}\right]-e_{1} \sigma,
\end{gathered}
$$

Owing to the fact that Eq. (4.14) is nonlinear only to second order, a potential solution has the form

$$
\begin{gathered}
U \approx U_{1} \exp (i \omega t)+U_{2} \exp (i 2 \omega t)+c . c . \\
\sigma \approx \sigma_{1}(\mathbf{r}) \exp (i \omega t)+\sigma_{2}(\mathbf{r}) \exp (i 2 \omega t)+\text { c.c. }
\end{gathered}
$$

where $\sigma_{1}$ and $\sigma_{2}$ refer to the linear and the nonlinear waves, respectively, $U_{1}$ and $U_{2}$ refer to the amplitude of the linear pulsation and the nonlinear response, respectively, $\mathbf{r}$ refers to the three-dimensional space coordinate position of the field point that may be expressed in the Cartesian coordinate as $\mathbf{r}=x_{1} \hat{i}+x_{2} \hat{j}+x_{3} \hat{k}$.

Substituting Eq. (4.15) into Eq. (14) and assuming that $1>U_{1}>U_{2}$ yield

$$
U_{1}=g_{1} \sigma_{1}, U_{2}=g_{2} \sigma_{2}+\Gamma \sigma_{1}^{2},
$$


where

$$
\begin{gathered}
g_{1}=-\frac{e_{1}}{\left(\omega_{1}^{2}-\omega^{2}\right)+i\left(\omega \delta_{1}+\omega^{3} R_{1} / c_{m}\right)}, g_{2}=-\frac{e_{1}}{\left(\omega_{1}^{2}-4 \omega^{2}\right)+i\left(2 \omega \delta_{1}+8 \omega^{3} R_{1} / c_{m}\right)}, \\
\Gamma=g_{1}^{2} \frac{\left(G_{1}-3 \omega^{2} H_{1}\right)+i \omega \delta_{2}}{\left(\omega_{1}^{2}-4 \omega^{2}\right)+i\left(2 \omega \delta_{1}+8 \omega^{3} R_{1} / c_{m}\right)}
\end{gathered}
$$

Expanding the displacement vector $\mathbf{u}$, up to second order approximation, as

$$
\mathbf{u}=\mathbf{u}_{1}(\mathbf{r}) \exp (i \omega t)+\mathbf{u}_{2}(\mathbf{r}) \exp (i 2 \omega t)+\text { c.c. },
$$

one obtains

$$
\sigma_{1}=\tilde{C}_{11}^{(1)} \nabla \cdot \mathbf{u}_{1}, \sigma_{2}=\tilde{C}_{11}^{(2)} \nabla \cdot \mathbf{u}_{2}
$$

If the size distribution function of the bubbles is specified as $n\left(R_{0}\right)$ (so that $n\left(R_{0}\right) d R_{0}$ is the number of bubbles with radii from $R_{0}$ to $R_{0}+d R_{0}$ in unit volume), the variation of the specific volume of the bubbles $V_{t}$ is related to the volume variation of an individual bubble $U$ by the relationship

$$
V_{t}=\int U n\left(R_{0}\right) d R_{0}
$$

Expanding $V_{t}$ to second order approximation as

$$
V_{t}=V_{1} \exp (i \omega t)+V_{2} \exp (i 2 \omega t)+\text { c.c. , }
$$

one obtains from Eqs. (4.16-4.19)

$$
V_{1}=V_{g 1} \tilde{C}_{11}^{(1)} \nabla \cdot \mathbf{u}_{1}, V_{2}=V_{g 2} \tilde{C}_{11}^{(2)} \nabla \cdot \mathbf{u}_{2}+V_{\Gamma}\left(\tilde{C}_{11}^{(1)} \nabla \cdot \mathbf{u}_{1}\right)^{2}
$$

where

$V_{g 1}=\int g_{1} n\left(R_{0}\right) d R_{0}, V_{g^{2}}=\int g_{2} n\left(R_{0}\right) d R_{0}$, and $V_{\Gamma}=\int \Gamma n\left(R_{0}\right) d R_{0}$.

If all the bubbles are of the uniform radius $R_{0}, V_{t}$ is related to $U$ by the relationship $V_{t}=N U$ with $N=3 \beta\left(4 \pi R_{0}^{3}\right)^{-1}$ being the number of bubbles in unit volume. In such cases one has

$$
V_{g 1}=N g_{1}, V_{g^{2}}=N g_{2}, V_{\Gamma}=N \Gamma \text {. }
$$

Expressing the volume change of the solid phase as $\theta^{s}=\nabla \cdot \mathbf{u}$, one may rewrite Eq. (4.12) as follows:

$$
\tilde{C}_{11}^{(1)} \nabla \cdot \mathbf{u}_{1}=C_{11}\left(\nabla \cdot \mathbf{u}_{1}-V_{1}\right), \tilde{C}_{11}^{(2)} \nabla \cdot \mathbf{u}_{2}=C_{11}\left(\nabla \cdot \mathbf{u}_{2}-V_{2}\right)
$$

\subsubsection{The wave equations}

According to Ref. [6], $\rho_{m} F_{1}$ is defined as the effective density of the soft medium surrounding the bubbles, the effective density of the effective medium may thus be 
identified as $\tilde{\rho}=\rho_{m} F_{1}(1-\beta)+\rho_{g} \beta$. Since the dynamic nonlinearity of the medium associated with the finite amplitude of wave has been ignored, the wave equation of the effective medium may be written as follows:

$$
\nabla\left(\tilde{C}_{11} \nabla \cdot \mathbf{u}\right)-\tilde{\mu} \nabla \times \nabla \times \mathbf{u}=\tilde{\rho} \frac{\partial^{2} \mathbf{u}}{\partial t^{2}}
$$

We represent the displacement vector $\mathbf{u}$ in terms of the sum of the potentials, as follows:

$$
\mathbf{u}=\nabla \Phi+\nabla \times \mathbf{\Psi}
$$

for which the vector potential $\Psi$ satisfies $\nabla \cdot \Psi=0$.

Up to second order approximation, the scalar potential $\Phi$ may be written as

$$
\Phi \approx \Phi^{(1)}+\Phi^{(2)}+\text { c.c. },
$$

where

$$
\Phi^{(1)}(\mathbf{r}, t)=\Phi_{1}(\mathbf{r}) \exp (i \omega t), \Phi^{(2)}(\mathbf{r}, t)=\Phi_{1}(\mathbf{r}) \exp (i 2 \omega t) .
$$

Substitution of Eqs. (4.23) and (4.24) in Eq. (4.22) yields

$$
\begin{gathered}
\tilde{C}_{11}^{(1)} \nabla^{2} \Phi^{(1)}=\tilde{\rho} \frac{\partial^{2} \Phi^{(1)}}{\partial t^{2}}, \tilde{C}_{11}^{(2)} \nabla^{2} \Phi^{(2)}=\tilde{\rho} \frac{\partial^{2} \Phi^{(2)}}{\partial t^{2}}, \\
\tilde{\mu} \nabla^{2} \Psi=\tilde{\rho} \frac{\partial^{2} \Psi}{\partial t^{2}} .
\end{gathered}
$$

As observed from Eq. (4.27), this equation takes on a non-resonant form and the influence of the existence of the bubbles on the propagation of the shear wave in a bubbly soft medium is insignificant. In the following we shall restrain our attention in the propagation of the compressional wave in such a medium.

Substituting Eqs. (4.20), (4.21), and (4.25) in Eq. (4.26), we arrive at the equations that must be satisfied by the scalar potentials of the first and the second order, as follows:

$$
\begin{gathered}
C_{11} \nabla^{2} \Phi_{1}+\tilde{\rho} \omega^{2}\left(1+V_{g 1} C_{11}\right) \Phi_{1}=0 \\
C_{11} \nabla^{2} \Phi_{2}+4 \tilde{\rho} \omega^{2}\left(1+V_{g 2} C_{11}\right) \Phi_{2}=C_{11} V_{\Gamma}\left(\tilde{C}_{11}^{(1)}\right)^{2}\left(\nabla^{2} \Phi_{1}\right)^{2},
\end{gathered}
$$

Eqs. (4.28a) and (4.28b) give description of the the propagation of the fundamental and the second harmonics of the compressional wave in a bubbly soft medium, respectively. Note that Eq. (4.28) is derived on the basis of Eq. (4.22) which is expressed as a form of a linear order terms with nonlinear propagation parameters due to the nonlinear oscillation of bubbles. Consequently it is seen that Eq. (4.28b) takes a simple form without any quadratic term involved that represents the dynamic nonlinearity caused by the finite amplitude of wave. In the right hand side of this equation, however, a quadratic term appears that accounts for the transfer of acoustical energy from the fundamental to the second harmonic waves, which results from the strong physical nonlinearity that dominates for a bubbly medium. 


\subsubsection{One-dimensional case}

Now consider a one-dimensional case in which a plane longitudinal wave propagates along the $\hat{x}_{1}$ direction in a bubbly soft medium. For simplicity while without losing generality, we assume that all the bubbles are of the same equilibrium radius $R_{0}$. In such a case Eq. (4.25) becomes

$$
\Phi^{(1)}\left(x_{1}, t\right)=\Phi_{1}\left(x_{1}\right) \exp (i \omega t), \Phi^{(2)}\left(x_{1}, t\right)=\Phi_{2}\left(x_{1}\right) \exp (i 2 \omega t) .
$$

Using the Kelvin-Voigt viscoelastic model, the Lamé coefficients of the soft viscoelastic medium may be rewritten as

$$
\lambda=\lambda_{m}, \mu=\mu_{m}+\eta_{m} \partial / \partial t .
$$

Substitution of Eqs. (4.29) and (4.30) in Eq. (4.28) yields

$$
\begin{gathered}
\frac{d^{2} \Phi_{1}}{d x_{1}^{2}}+\left(\Lambda_{1}^{2}+\tilde{\rho} \omega^{2} V_{g 1}\right) \Phi_{1}=0, \\
\frac{d^{2} \Phi_{2}}{d x_{1}^{2}}+\left(\Lambda_{2}^{2}+4 \tilde{\rho} \omega^{2} V_{g^{2}}\right) \Phi_{2}=\tilde{\rho}^{2} \omega^{4} V_{\Gamma} \Phi_{1}^{2},
\end{gathered}
$$

where $\Lambda_{1}^{2}=\omega^{2} \tilde{\rho} /\left[\left(\lambda_{m}+2 \mu_{m}\right)+i 2 \omega \eta_{m}\right]$, and $\Lambda_{2}^{2}=4 \omega^{2} \tilde{\rho} /\left[\left(\lambda_{m}+2 \mu_{m}\right)+i 4 \omega \eta_{m}\right]$.

We introduce the effective wave numbers defined as complex numbers that can be expressed in terms of real effective wave speeds and effective attenuations, as follows:

$$
\tilde{k}_{1}=\sqrt{\Lambda_{1}^{2}+\tilde{\rho} \omega^{2} V_{g 1}}=\omega / c_{1}-i \alpha_{1}, \tilde{k}_{2}=\sqrt{\Lambda_{2}^{2}+4 \tilde{\rho} \omega^{2} V_{g 2}}=2 \omega / c_{2}-i \alpha_{2} ，
$$

where $\tilde{k}_{i}$ refers to the effective wave numbers, $c_{i}$ and $\alpha_{i}$ refer to the (real) effective wave speed and the effective attenuation, respectively; and the subscripts $i=1,2$ refer to the fundamental wave and the second harmonic wave, respectively.

Assuming $\Phi_{1}=\Phi_{1 A} \exp \left(-i \tilde{k}_{1} x_{1}\right)$, from Eq. (4.31a) one readily obtains the expressions of $c_{1}$ and $\alpha_{1}$, as follows:

$$
c_{1}=\left(\frac{-A_{1}+\sqrt{A_{1}^{2}+B_{1}^{2}}}{2}\right)^{-1 / 2}, \alpha_{1}=\omega B_{1} c_{1} / 2 .
$$

where the parameters of $A_{2}, B_{2}$, and $C$ are given as follows:

$$
\begin{aligned}
& A_{1}=\tilde{\rho} \omega^{2}\left[\frac{\lambda_{m}+2 \mu_{m}}{\left(\lambda_{m}+2 \mu_{m}\right)^{2}+4 \omega^{2} \eta_{m}^{2}}-\frac{N e_{1}\left(\omega_{1}^{2}-\omega^{2}\right)}{\left(\omega_{1}^{2}-\omega^{2}\right)^{2}+\left(\omega \delta_{1}+\omega^{3} R_{1} / c_{m}\right)^{2}}\right], \\
& B_{1}=\tilde{\rho} \omega^{2}\left[\frac{2 \omega \eta_{m}}{\left(\lambda_{m}+2 \mu_{m}\right)^{2}+4 \omega^{2} \eta_{m}^{2}}-\frac{N e_{1}\left(\omega \delta_{1}+\omega^{3} R_{1} / c_{m}\right)}{\left(\omega_{1}^{2}-\omega^{2}\right)^{2}+\left(\omega \delta_{1}+\omega^{3} R_{1} / c_{m}\right)^{2}}\right] .
\end{aligned}
$$

It is apparent that the solution of Eq. (4.31b) is supposed to consist of a general solution and a special solution. By invoking the boundary condition that the second harmonic wave 
should be zero at the beginning, i.e., $\left.\Phi_{2}\right|_{x_{1}=0}=0$, one can readily determine the expression of the second harmonic wave as follows:

$$
\Phi_{2 g}=C \Phi_{1 A}^{2}\left[\exp \left(-i \tilde{k}_{2} x\right)-\exp \left(-i \tilde{k}_{2} x\right)\right]+\text { c.c. },
$$

where

$$
c_{2}=\left(\frac{-A_{2}+\sqrt{A_{2}^{2}+B_{2}^{2}}}{2}\right)^{-1 / 2}, \alpha_{2}=\omega B_{2} c_{2} .
$$

where the parameters of $A_{2}, B_{2}$ and $C$ are given as follows:

$$
\begin{gathered}
A_{2}=\tilde{\rho} \omega^{2}\left[\frac{\lambda_{m}+2 \mu_{m}}{\left(\lambda_{m}+2 \mu_{m}\right)^{2}+16 \omega^{2} \eta_{m}^{2}}-\frac{N e_{1}\left(\omega_{1}^{2}-4 \omega^{2}\right)}{\left(\omega_{1}^{2}-4 \omega^{2}\right)^{2}+\left(2 \omega \delta_{1}+8 \omega^{3} R_{1} / c_{m}\right)^{2}}\right], \\
B_{2}=\tilde{\rho} \omega^{2}\left[\frac{4 \omega \eta_{m}}{\left(\lambda_{m}+2 \mu_{m}\right)^{2}+16 \omega^{2} \eta_{m}^{2}}-\frac{N e_{1}\left(2 \omega \delta_{1}+8 \omega^{3} R_{1} / c_{m}\right)}{\left(\omega_{1}^{2}-4 \omega^{2}\right)^{2}+\left(2 \omega \delta_{1}+8 \omega^{3} R_{1} / c_{m}\right)^{2}}\right] . \\
C=\left[\left(M_{1} N_{1}+M_{2} N_{2}\right)+i\left(M_{2} N_{1}-M_{1} N_{2}\right)\right] /\left(N_{1}^{2}+N_{2}^{2}\right),
\end{gathered}
$$

where

$$
\begin{gathered}
M_{1}=\tilde{\rho}^{2} \omega^{4} N e_{1}^{2}\left[\left(G_{1}-3 \omega^{2} H_{1}\right)\left(A_{1}^{2}-B_{1}^{2}\right)+2 A_{1} B_{1} \omega \delta_{2}\right], \\
M_{2}=\tilde{\rho}^{2} \omega^{4} N e_{1}^{2}\left[\left(A_{1}^{2}-B_{1}^{2}\right) \omega \delta_{2}-2 A_{1} B_{1}\left(G_{1}-3 \omega^{2} H_{1}\right)\right], \\
N_{1}=K_{1}\left(-4 A_{1}+A_{2}\right)+L_{1}\left(B_{2}-4 B_{1}\right), \\
N_{2}=L_{1}\left(-4 A_{1}+A_{2}\right)-K_{1}\left(B_{2}-4 B_{1}\right),
\end{gathered}
$$

where

$$
\begin{gathered}
K_{1}=\left(\omega_{1}^{2}-4 \omega^{2}\right)\left[\left(\omega_{1}^{2}-\omega^{2}\right)^{2}-\left(\omega \delta_{1}+\omega^{3} R_{1} / c_{m}\right)^{2}\right] \\
-2\left(\omega_{1}^{2}-\omega^{2}\right)\left(\omega \delta_{1}+\omega^{3} R_{1} / c_{m}\right)\left(2 \omega \delta_{1}+8 \omega^{3} R_{1} / c_{m}\right), \\
L_{1}=\left[\left(\omega_{1}^{2}-\omega^{2}\right)^{2}-\left(\omega \delta_{1}+\omega^{3} R_{1} / c_{m}\right)^{2}\right]\left(2 \omega \delta_{1}+8 \omega^{3} R_{1} / c_{m}\right) \\
+2\left(\omega_{1}^{2}-4 \omega^{2}\right)\left(\omega_{1}^{2}-\omega^{2}\right)\left(\omega \delta_{1}+\omega^{3} R_{1} / c_{m}\right) .
\end{gathered}
$$

In practical, the nonlinearity parameter $(B / A)$ is of particular significance that may be used to define the nonlinearity of the media. In the present study, therefore, we introduce an effective nonlinearity parameter $(B / A)_{e}$ to describe the extent to which the nonlinearity of a bubbly medium is enhanced by the nonlinear oscillation of bubbles. The value of $(B / A)_{e}$ may be determined near the natural frequency of bubble, as given below: [39] 


$$
(B / A)_{e}=\left|\frac{4 C \rho_{m} c_{m}^{3}\left(2 \alpha_{1}-\alpha_{2}\right)}{\omega}-2\right|
$$

It is apparent that the expression of the effective nonlinearity parameter $(B / A)_{e}$ derived here is identical in form with the one obtained by Ma et al except that their approach only applies to a liquid containing shelled bubbles [39].

Despite the similarity between the EMM and other methodologies which also investigate the wave propagation in inhomogeneities by treating the media as a homogeneous effective medium [40-45], the EMM definitely differs from them in several respects. It is a fundamental distinction that the EMM accounts for the nonlinearity of the bubbly soft medium up to a second-order approximation, whereas most of the previous ones only use linear approximation when homogenizing the medium [40-43], which inevitably loses significant details for bubbly soft media with particularly strong "physical" nonlinearity $[3,4]$. Second, the EMM permits one to take into consideration the effects of weak compressibility, surface tension, viscosity, surrounding pressure, and an encapsulating elastic shell, which can only be partially accounted for by other methods [44,45]. There are important practical reasons for pursuing more precise results in various engineering situations, for which the incorporation of these effects is apparently necessary. (For a detailed discussion on this topic and a comparison between the application of the EMM and some other methods in different cases serving as simple models of practical situations, see Ref. [12]) Finally, the EMM could apply to three-dimensional cases rather than onedimensional cases. Most of the relative studies investigate only the wave propagation in an infinite effective medium for which the one-dimensional approximation is sufficient, but it is indispensable to obtain the three-dimensional effective parameters for some practical structures of finite sizes.

It must be stressed, however, that there also exist limitations of the application of the EMM despite its effectiveness. First, the multiple scattering effects have been neglected when we homogenizing the bubbly soft medium, therefore the EMM can not apply to bubbly media with extremely large volume fractions. Second, the EMM is developed under quadratic approximation by employing a simple perturbation approach, and the nonlinearity of medium is studied by inspecting the second harmonic wave with no harmonics of orders higher than 2 involved. Finally, the present model could not enable full incorporation of all the practical effects that affect the acoustical properties of a bubbly medium, such as the buckling of bubbles [46]. These problems will be the focus of a future study.

\section{Optimal acoustic attenuation of bubbly soft media}

In this section, we present an optimization method on the basis of fuzzy logic (FL) and genetic algorithm (GA) to obtain the optimal acoustic attenuation of a longitudinal wave in a bubbly soft medium by optimizing the parameters of size distribution of bubbles. This optimization method can be used to design acoustic absorbent with uniformly high acoustic attenuation within the frequency band of interest, without the precise mathematical model required.

\subsection{Acoustic attenuation in bubbly soft media}

The oscillation of an air bubble in a soft medium is special, due to the fact that only if the ratio $\lambda / \mu$ is sufficiently large can this bubble behave effectively as a resonant oscillator [7]. When compared with the viscoelasticity of the medium, the resonance of the system 
introduced by bubbles becomes the most dominant mechanism for acoustic attenuation [13]. For a bubbly soft medium, it is apparent that the acoustic properties are affected by all the structural parameters, of the bubbles and of the medium. By employing the EMM presented in Section 4, we can accurately predict the acoustic parameters of a bubbly soft medium for arbitrary structural parameters. In this situation, one may expect to enhance the acoustic attenuation of such a medium in an optimal manner with the aid of a fast computer.

Consider the one-dimensional propagation of a longitudinal wave in an infinite bubbly soft medium with small volume fraction $\Phi_{\mathrm{b}}$. On condition that the bubbles are not very densely packed, the multiple scattering effects are negligible, and the acoustic properties of such a bubbly soft medium can be described by using the EMM.

If all the bubbles are of uniform radius $r_{0}$, the bubble volume fraction will be $\Phi_{\mathrm{b}}=4 \pi N_{\mathrm{b}}\left(r_{0}\right)^{3} / 3$ with $N_{\mathrm{b}}$ being the number of bubbles per unit volume. When the bubble sizes are not uniformly distributed, the volume fraction is related to the distribution function $n(r)$ of bubble sizes, as follows:

$$
\Phi_{\mathrm{b}}=4 \pi \int_{0}^{\infty} n(r) r^{3} d r / 3
$$

where $n(r) d r$ is the number of bubbles per unit volume having a radius between $r$ and $r+d r$. For simplicity, the bubbles in the soft medium are assumed to be free bubbles (no encapsulating shells), the effects of surface tension and the ambient pressure are neglected, and acoustic nonlinearity of the bubbly soft medium are not taken into account. Then the effective acoustic attenuation of longitudinal wave in the bubbly soft medium can be derived from Eq. (4.32), as follows:

$$
\alpha_{1}^{\prime}=A_{1}^{\prime}\left(-B_{1}^{\prime} / 2+\sqrt{B_{1}^{\prime 2} / 4+A_{1}^{\prime}}\right)^{-1 / 2},
$$

where the parameters of $A$ and $B$ are given as follows:

$$
\begin{gathered}
B_{1}^{\prime}=\int_{0}^{\infty} \frac{n(r) e(r) \rho_{0}}{\chi_{1}(r)}\left(\cos \phi(r)-\frac{2 \mu_{v} \omega \sin \phi(r)}{\lambda+2 \mu}\right) d r+\frac{4 \mu_{v} \omega A_{1}^{\prime}-\omega^{2} \rho_{0}}{\lambda+2 \mu}, \\
A_{1}^{\prime}=\int_{0}^{\infty} \frac{n(r) e(r) \rho_{0} \sin \phi(r)}{2 \chi_{1}(r)} d r+\frac{2 \mu_{v} \omega^{3} \rho_{0}}{2(\lambda+2 \mu)+8 \mu_{v}^{2} \omega^{2}}
\end{gathered}
$$

where

$$
\chi_{1}(r)=\left[\left(1-\frac{\omega_{0}^{2}}{\omega^{2}}\right)^{2}+\left(\frac{\omega r}{c_{l}}+\frac{4 \mu_{v}}{\omega \rho_{0} r^{2}}\right)^{2}\right]^{1 / 2}, \phi(r)=\tan ^{-1}\left[\left(\frac{4 \mu_{v}}{\omega \rho_{0} r^{2}}+\frac{\omega r}{c_{l}}\right)\left(1-\frac{\omega_{0}^{2}}{\omega^{2}}\right)^{-1}\right],
$$

where $\rho_{0}$ is the mass density, $\mu_{v}$ is the lossy factor given by the Kelvin-Voigt viscoelastic model, $c_{l}$ is the velocity of the longitudinal elastic wave.

In general, the enhancement of acoustic attenuation is equivalent to regularly providing sufficient acoustic attenuation in the frequency range of interest. Due to the resonance of the system introduced by bubbles, the acoustic attenuations exhibit a remarkable enhancement effect near the bubble resonant frequencies, and there exist resonance peaks in the spectral domain [40]. We consider the acoustic attenuation caused by the oscillation of the bubbles, 
and neglect the contribution of the viscosity to the acoustic attenuation. The resonance frequency can be decreased by reducing the shear modulus of the medium or enlarging bubbles, and the acoustic attenuation will be collectively enhanced as $\Phi_{b}$ increases [45]. However, it is impractical to unlimitedly increase the volume fraction and dimension of bubbles. The strength of the bubbly medium will be weakened if the bubbles are too densely packed or oversize, and oversize bubbles are not feasible for a practical medium of finite size. It is of interest to provide regularly high acoustic attenuation in targeted frequency range while minimizing the volume fraction and dimension of bubbles.

To decrease the resonance location, it is more effective to reduce the shear modulus of the medium than to merely enlarge bubbles. Besides, the acoustic attenuation will also be enhanced as $\Phi_{b}$ remains constant while the shear modulus reduces [45]. Hence we choose silicone that has low shear modulus as the medium for which the mechanical parameters are: $\rho_{0}=1000 \mathrm{~kg} / \mathrm{m}^{3}$, the velocity of the longitudinal and the shear elastic wave are $c_{l}=1700 \mathrm{~m} / \mathrm{s}$ and $c_{s}=20 \mathrm{~m} / \mathrm{s}$, respectively [47]. The lossy factor is chosen as $\mu_{v}=80 \mathrm{P}$. On the other hand, it has been proved that the nonuniform distribution of bubble sizes has an averaging effect tends to increase the acoustic attenuation over a wider frequency range and result in a much broader resonance peak [40]. In what follows, therefore, distribution of bubble sizes is introduced and the probability density function of normal distribution is employed to describe the distribution function. Due to the peak-broadening effects of size distribution, together with the amplitude-enhancing effects of volume fraction, one may hope to obtain an optimal acoustic attenuation for a bubbly soft medium by choosing the structural parameters appropriately. This leads to the necessity of some optimization method.

For such a problem with multiple adjustable parameters, a full-space search method will not be practical, and a global optimization method is expected to be effective [48]. The success of an optimization method depends to a great extent on the definition of a proper objective function. For such a problem, however, it may be difficult to mathematically create an appropriate objective function in traditional ways, since the ability of acoustic attenuation of a medium is usually evaluated qualitatively. With the purpose of avoiding such mathematical efforts, we will define the objective function by using FL that bases on decision rules rather than mathematical equations and describe linguistically the relationship between input and output $[49,50]$, and use a GA that can locate the global optimum despite that the objective function is built without knowing its clear mathematical model [51-53].

\subsection{Numerical example}

In the following we will exemplify a numerical case for enhancing the acoustic attenuation of the bubbly soft medium in an optimal manner. As an example, we intend to obtain uniformly effective acoustic attenuation for longitudinal wave propagating within the bubbly soft medium, in a broad frequency range at intermediate frequencies. And the following requirement is proposed:

1. The bubbly medium can attenuate longitudinal wave by no less than $10 \mathrm{~dB} / \mathrm{cm}$, in a frequency range as broad as possible within the intermediate frequency range of $[5 \mathrm{KHz}$, $800 \mathrm{KHz}]$.

2. The wave at the frequency of $5 \mathrm{KHz}$ should be effectively attenuated.

3. The acoustic attenuations in targeted frequency range must be uniform. 
This quantitative requirement serves for the goal of the optimization. The effectiveness of optimization method will be eventually evaluated in terms of the extent to which the requirement is fulfilled.

For a particular medium, the large and the small bubbles contribute to the acoustic attenuation at low and high frequencies, respectively. It is thus possible to increase acoustic attenuation at low frequencies as well as extend the width of resonance peak, by introducing the size distribution of large and small bubbles and tuning up their parameters properly. Then the distribution function $n(r)$ is given as below:

$$
n(r)=\left\{\begin{array}{rl}
n_{1}(r)+n_{2}(r), & r_{L} \leq r \leq r_{U} \\
0, & \text { elsewhere }
\end{array},\right.
$$

where $r_{U}\left(r_{L}\right)$ refers to the radius of the largest (smallest) bubble in the medium, $n_{1}(r)$ and $n_{2}(r)$ refer to the number densities of large and small bubbles respectively, as follows:

$$
n_{1}(r)=n_{0} R_{\mathrm{b}}\left(r_{2} / r_{1}\right)^{3} \exp \left[-\left(r / r_{1}-1\right) /\left(2 \sigma_{\mathrm{b} 1}\right)\right]^{2}, n_{2}(r)=n_{0} \exp \left[-\left(r / r_{2}-1\right) /\left(2 \sigma_{\mathrm{b} 2}\right)\right]^{2},
$$

where the value of $R_{\mathrm{b}}\left(r_{2} / r_{1}\right)^{3}$ represents the ratio of number density of large bubbles to small bubbles, $r_{j}$ and $\sigma_{\mathrm{bj}}(j=1,2)$ refers to the center and the width of size distribution, respectively. The value of $n_{0}$ can be easily determined from the relationship given by Eq.(5.2). Now the bubble parameters are the only adjustable parameters affecting the acoustic attenuation of the bubbly medium, including $\Phi_{\mathrm{b}}$ and the parameters of distribution function $n(r)$. It is apparent that the objective function to be created is a multiple inputs problem and the input variables consist of all these adjustable parameters, i.e., $\Phi_{\mathrm{b}}, r_{L, U}, r_{1,2}, R_{\mathrm{b}}, \sigma_{\mathrm{b} 1,2}$.

It is obvious that the ability of acoustic attenuation of the bubbly medium mostly depends on the location and shape of the lowest resonance peak in spectral domain. To describe the location and width of this resonance peak, we introduce two parameters $f_{0}=f_{L}$ and $W_{b}=f_{U}-f_{L}$ defined as the lowest effective attenuation frequency and the effective attenuation bandwidth, respectively. Here $f_{U}\left(f_{L}\right)$ is the upper (lower) limit of a frequency band within which acoustic attenuation of any frequency is more than a threshold value $\alpha_{t}\left(\alpha_{t}=10 \mathrm{~dB} / \mathrm{cm}\right)$, and here does not exist a $f_{L}^{\prime}<f_{L}$, such that $f_{L}^{\prime}$ satisfies the condition as well. And a standard deviation function $\Sigma$ is introduced to scale the degree of regularity of attenuation, as follows:

$$
\Sigma=\sqrt{\int_{f_{L}}^{f_{U}}\left[\alpha_{1}^{\prime}(f)-\alpha_{t}\right]^{2} d f /\left(f_{U}-f_{L}\right)},
$$

where $\alpha_{1}^{\prime}(f)$ refers to acoustic attenuation at the frequency of $f$. It is apparent that the introduced parameters $f_{0}, W_{b}$ and $\Sigma$ can be easily obtained from the acoustic attenuation predicted by EMM and describe quantitatively the characteristic of the lowest resonance peak in spectral domain.

By using FL, we set up a fuzzy inference system (FIS), for which the parameters $f_{0}, W_{b}$ and $\Sigma$ are chosen as the input parameters and the explicit output is defined as $s(0 \leq s \leq 100)$.

There are three membership functions for $f_{0}$ : "low", "intermediate" and "high". And there are three membership functions for $W_{b}$ as well: "narrow", "average" and "broad". Similarly $\Sigma$ consists of three conditions of degree of deviation denoted by "small", "ordinary" and "large". The membership functions of the inputs are built on a simple Gaussian curve due to its smoothness in varying. 
Three inputs are captured consisting of $f_{0}, W_{b}$ and $\sigma$, and the fuzzy relation between the fuzzy inputs and the required output $s$ are shown by the following inference rules:

Rules 1: If $\left(f_{0}\right.$ is "high") or ( $W_{b}$ is "narrow") and ( $\sigma$ is "large") then ( $s$ is "bad")

Rules 2: If ( $f_{0}$ is "intermediate") and ( $W_{b}$ is "average") and ( $\sigma$ is "ordinary") then ( $s$ is "mediocre")

Rules 3: If ( $f_{0}$ is "low") and ( $W_{b}$ is "average") and ( $\sigma$ is "ordinary") then (s is "good")

Rules 4: If $\left(f_{0}\right.$ is "intermediate") and $\left(W_{b}\right.$ is "broad") and ( $\sigma$ is "ordinary") then (s is "good")

Rules 5: If $\left(f_{0}\right.$ is "intermediate") and ( $W_{b}$ is "average") and ( $\sigma$ is "small") then ( $s$ is "good")

Rules 6: If $\left(f_{0}\right.$ is "intermediate") and ( $W_{b}$ is "broad") and ( $\sigma$ is "small") then (s is "very good")

Rules 7: If $\left(f_{0}\right.$ is "low") and ( $W_{b}$ is "average") and ( $\sigma$ is "small") then ( $s$ is "very good")

Rules 7: If ( $f_{0}$ is "low") and ( $W_{b}$ is "broad") and ( $\sigma$ is "ordinary") then ( $s$ is "very good")

Rules 8: If $\left(f_{0}\right.$ is "low") and ( $W_{b}$ is "broad") and ( $\sigma$ is "small") then (s is "excellent")

The above inference rules relate these inputs to the output $s$ consisting of five membership functions: "bad", "mediocre", "good", "very good", "excellent". The triangular membership function is adopted because this membership representation shows boundary clearly.

It is apparent that the mapping of the multiple input parameters $\left(f_{0}, W_{b}\right.$ and $\left.\Sigma\right)$ to the output $s$ can be conveniently constructed, by defining the fuzzy rules as a set of linguistic rules according to the aforementioned requirement, without knowing the clear mathematical model. Then the value of output $s$ gives a quantitative description of the extent to which the qualitative requirement is met. A bubbly soft medium of better acoustic attenuation will correspond to an output of larger value. With the aid of the FIS, we readily define an objective function corresponding to this nine-input, one-output problem. Mathematically speaking, this objective function based on FL may not be completely precise, and the clear mathematical model is not visible. But it is readily guaranteed that the acoustic attenuation ability of a bubbly soft medium is evaluated strictly by the decision rules, which is the unique advantage of FL for such a problem.

By defining an objective function for mapping the multiple inputs properly to a clear output, the optimal enhancement of acoustic attenuation ability amounts to an optimization problem of generating a maximal output by tuning up the inputs. Such an optimization is performed by employing GA optimizer. The objective function and the output $s$ are regarded as the fitness function and the fitness, respectively. The nine input variables are encoded as the chromosome. GA optimizer searches for the optimum of fitness function by adjusting the bubble parameters and seeking the most proper proportion. To guarantee the physical feasibility, a set of constraints of the variables are applied, as follows:

$$
0<\Phi_{\mathrm{b}} \leq 5 \%, 10 \mu \mathrm{m} \leq r_{L} \leq r_{1} \leq r_{2} \leq r_{U} \leq 2 \mathrm{~mm}, 0<R_{\mathrm{b}} \leq 10,0<\sigma_{\mathrm{b} 1}, \sigma_{\mathrm{b} 2} \leq 1 .
$$

In the process of GA optimization, the number of population and maximal number of generation are chosen as 80 and 500, respectively, the crossover and mutation ratio are set to 0.8 and 0.05 , respectively [53].

As a result, the bubbly silicone with optimized structural parameters has a value of fitness as high as 99.2. Correspondingly, the lower and upper limits of the effective attenuation band are $f_{L} \approx 5 \mathrm{KHz}$ and $f_{u} \approx 800 \mathrm{KHz}$, respectively. Then one has $f_{0} \approx 5 \mathrm{KHz}$ and $W_{b} \approx 795 \mathrm{KHz}$. The optimal acoustic attenuation versus frequency is plotted in Fig. 5.1. Figure 5.2 displays the 
corresponding size distribution function $n(r)$. The numerical result shows that the goal of optimization is attained perfectly, which is indicated numerically by the fitness and illustrated graphically in Fig. 5.1. The bubbly medium with optimized structural parameters can effectively attenuate longitudinal waves in an intermediate frequency range of $[5 \mathrm{KHz}$, $800 \mathrm{KHz}]$ with an acoustic attenuation approximating a constant value of $10(\mathrm{~dB} / \mathrm{cm})$. As shown by the results of the optimization process, such a bubbly medium may be applied to design broadband acoustic absorbent at intermediate frequencies with high efficiency. Compared with acoustic absorbent designed by using traditional method, the acoustic absorbent designed by using the present optimization method has broader attenuation band and higher efficiency. Moreover, the width and the location of its attenuation band may be conveniently controlled due to the adjustability of the objective function and the optimizer.

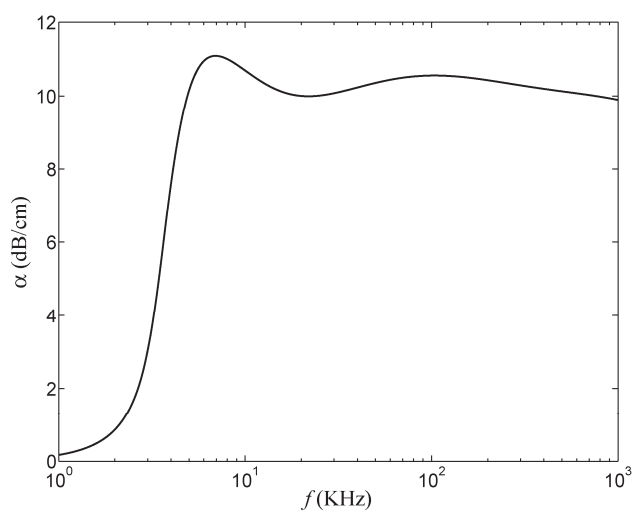

Fig. 5.1. Optimal acoustic attenuation $\alpha$ versus frequency for the bubbly silicone.

To study the necessity and efficiency of the optimization method, we also consider other three cases with no optimization applied. The structural parameters of these four cases are listed in Table I and the comparison of attenuation curves are displayed in Fig. 5.3. Observation of Fig. 5.3 and Table 5.1 shows that: (1) Case 4 has a better acoustic attenuation and a higher value of fitness than any of the other cases, which means that the relative values of fitness describe effectively the acoustic attenuation of the bubbly medium in accord with the requirement. (2) Size distribution effect helps to ameliorate the acoustic attenuation, which is proved by comparing case 3 with cases 1 and 2 . But the comparison between cases 3 and 4 shows that optimal acoustic attenuation can not be guaranteed by size distribution with random parameters. (3) The optimization method is efficient and essential in enhancing the acoustic attenuation of the bubbly medium.

\begin{tabular}{cccccccccc}
\hline Case & $\Phi_{\mathrm{b}}(\%)$ & $r_{L}(\mu \mathrm{m})$ & $r_{U}(\mathrm{~mm})$ & $r_{1}(\mathrm{~mm})$ & $r_{2}(\mu \mathrm{m})$ & $R_{\mathrm{b}}$ & $\sigma_{b 1}$ & $\sigma_{\mathrm{b} 2}$ & $s$ \\
\hline 1 & 1.4 & 1.7 & 1.7 & $\square$ & $\square$ & $\square$ & $\square$ & $\square$ & 39.6 \\
\hline 2 & 1.4 & 23 & 23 & $\square$ & $\square$ & $\square$ & $\square$ & $\square$ & 41.2 \\
\hline 3 & 0.5 & 40 & 1.1 & 0.5 & 200 & 0.08 & 0.4 & 0.1 & 63.6 \\
\hline 4 & 1.4 & 17 & 1.8 & 1.7 & 23 & 1.2 & 0.7 & 0.5 & 99.2 \\
\hline
\end{tabular}

Table 5.1 The structural parameters of the four different cases. 

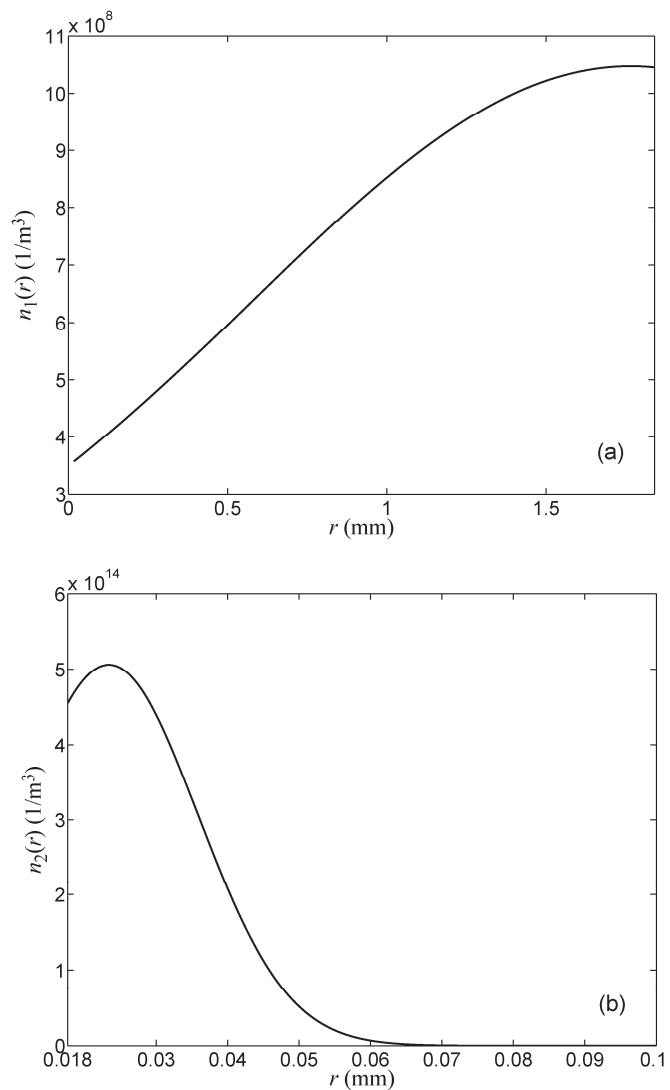

Fig. 5.2. Number densities of large (a) and small (b) bubbles in the bubbly silicone with optimal acoustic attenuation.

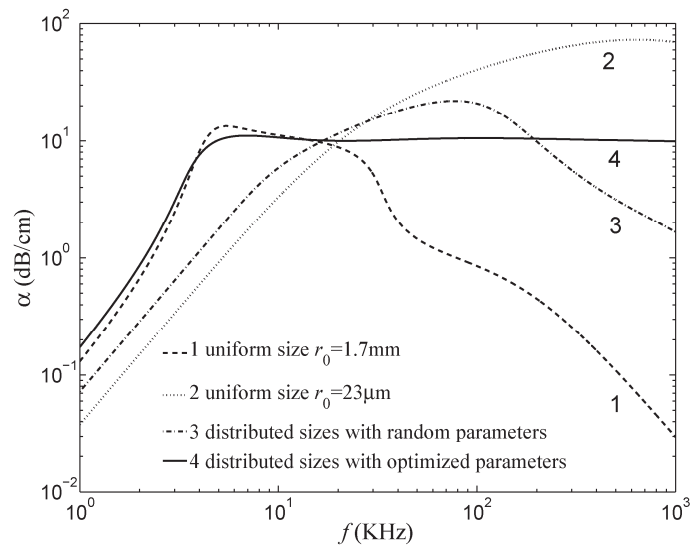

Fig. 5.3. Comparison of acoustic attenuations versus frequency for the four different cases. 


\section{Conclusions}

In this chapter, we first consider the acoustic propagation in a finite sample of bubbly soft elastic medium and solve the wave field rigorously by incorporating all multiple scattering effects. The energy converted into shear wave is numerically proved negligible as the longitudinal wave is scattered by the bubbles. Under proper conditions, the acoustic localization can be achieved in such a class of media in a range of frequency slightly above the resonance frequency. Based on the analysis of the spatial correlation characteristic of the wave field, we present a method that helps to discern the phenomenon of localization in a unique manner. Then we taken into consideration the effect of viscosity of the soft medium and investigate the localization in a bubbly soft medium by inspecting the oscillation phases of the bubble. The proper analysis of the oscillation phases of bubbles is proved to be a valid approach to identify the existence of acoustic localization in such a medium in the presence of viscosity, which reveals the existence of the significant phenomenon of phase transition characterized by an unusual collective behavior of the phases.

For infinite sample of bubbly soft medium, we present an EMM which enables the investigation of the strong nonlinearity of such a medium and accounts for the effects of weak compressibility, viscosity, surrounding pressure, surface tension, and encapsulating shells. Based on the modified equation of bubble oscillation, the linear and the nonlinear wave equations are derived and solved for a simplified 1-D case. Based on the EMM which can be used to conveniently obtain the acoustic parameters of bubbly soft media with arbitrary structural parameters, we present an optimization method for enhancing the acoustic attenuation of such media in an optimal manner, by applying FL and GA together. A numerical simulation is presented to manifest the necessity and efficiency of the optimization method. This optimization method is of potential application to a variety of situations once the objective function and optimizer are adjusted accordingly.

\section{References}

[1] E. Meyer, K. Brendel, and K. Tamm, J. Acoust. Soc. Am. 30, 1116 (1958).

[2] A. C. Eringen and E. S. Suhubi, Elastodynamics (Academic, New York, 1974).

[3] L. A. Ostrovsky, Sov. Phys. Acoust. 34, 523 (1988).

[4] L. A. Ostrovsky, J. Acoust. Soc. Am. 90, 3332 (1991).

[5] S. Y. Emelianov, M. F. Hamilton, Yu. A. Ilinskii, and E. A. Zabolotskaya, J. Acoust. Soc. Am. 115, 581 (2004).

[6] E. A. Zabolotskaya, Yu. A. Ilinskii, G. D. Meegan, and M. F. Hamilton, J. Acoust. Soc. Am. 118, 2173 (2005).

[7] L. D. Landau and E. M. Lifshits, Theory of Elasticity (Pergamon, Oxford, 1986).

[8] B. Liang and J. C. Cheng, Phys. Rev. E. 75, 016605 (2007).

[9] B. Liang, Z. M. Zhu, and J. C. Cheng, Chin. Phys. Lett. 23, 871 (2006).

[10] B. Liang, X. Y. Zou, and J. C. Cheng, Chin. Phys. Lett. 26, 024301 (2009).

[11] B. Liang, X. Y. Zou, and J. C. Cheng, Chin. Phys. B 19, 094301 (2010).

[12] B. Liang, X. Y. Zou, and J. C. Cheng, J. Acoust. Soc. Am. 124, 1419 (2008).

[13] G. C. Gaunaurd and J. Barlow, J. Acoust. Soc. Am. 75, 23 (1984).

[14] B. Liang, X. Y. Zou, and J. C. Cheng, Chin. Phys. Lett. 24, 1607 (2007). 
[15] K. X. Wang and Z. Ye, Phys. Rev. E 64, 056607 (2001).

[16] C. F. Ying and R. Truell, J. Appl. Phys. 27, 1086 (1956).

[17] G. C. Gaunaurd, K. P. Scharnhorst and H. Überall, J. Acoust. Soc. Am. 65, 573 (1979).

[18] D. M. Egle, J. Acoust. Soc. Am. 70, 476 (1981).

[19] R. L. Weaver, J. Acoust. Soc. Am. 71, 1608 (1982).

[20] K. Busch, C. M. Soukoulis, and E. N. Economou, Phys. Rev. B 50, 93 (1994).

[21] A. A. Asatryan, P. A. Robinson, R. C. McPhedran, L. C. Botten, C. Martijin de Sterke, T. L. Langtry, and N. A. Nicorovici, Phys. Rev. E 67, 036605 (2003).

[22] S. Gerristsen and G. E. W. Bauer, Phys. Rev. E 73, 016618 (2006).

[23] C. A. Condat, J. Acoust. Soc. Am. 83, 441 (1988).

[24] Z. Ye and A. Alvarez, Phys. Rev. Lett. 80, 3503 (1998).

[25] S. Catheline, J. L. Gennisson, and M. Fink, J. Acoust. Soc. Am. 114, 3087 (2003).

[26] Z. Fan, J. Ma, B. Liang, Z. M. Zhu, and J. C. Cheng, Acustica 92, 217 (2006).

[27] I. Y. Belyaeva and E. M. Timanin, Sov. Phys. Acoust. 37, 533 (1991).

[28] Z. Ye, H. Hsu, and E. Hoskinson, Phys. Lett. A 275, 452 (2000).

[29] A. Alvarez, C. C. Wang, and Z. Ye, J. Comp. Phys. 154, 231 (1999).

[30] L. L. Foldy, Phys. Rev. 67, 107 (1945).

[31] A. Ishimaru, Wave Propagation and Scattering in Random Media (Academic press, New York, 1978).

[32] B. C. Gupta and Z. Ye, Phys. Rev. E 67, 036606 (2003).

[33] M. Rusek, A. Orlowski, and J. Mostowski, Phys. Rev. E 53, 4122 (1996).

[34] A. Alvarez and Z. Ye, Phys. Lett. A 252, 53 (1999).

[35] C. C. Church, J. Acoust. Soc. Am. 97, 1510 (1995).

[36] C. H. Kuo, K. K. Wang and Z. Ye, Appl. Phys. Lett. 83, 4247 (2003).

[37] H. J. Feng and F. M. Liu, Chin. Phys. B 18, 1574 (2009).

[38] M. Mooney, J. Appl. Phys. 11, 582 (1940).

[39] J. Ma, J. F. Yu, Z. M. Zhu, X. F. Gong, and G. H. Du, J. Acoust. Soc. Am. 116, 186 (2004).

[40] G. C. Gaunaurd, H. Überall, J. Acoust. Soc. Am. 71, 282 (1982).

[41] G. C. Gaunaurd and W. Wertman, J. Acoust. Soc. Am. 85, 541 (1989).

[42] A. M. Baird, F. H. Kerr, and D. J. Townend, J. Acoust. Soc. Am. 105, 1527 (1999).

[43] D. G. Aggelis, S. V. Tsinopoulos, and D. Polyzos, J. Acoust. Soc. Am. 116, 3343 (2004).

[44] B. Qin, J. J. Chen, and J. C. Cheng, Acoust. Phys. 52, 490 (2006).

[45] B. Liang B, Z. M. Zhu, and J. C. Cheng, Chin. Phys. 15, 412 (2006).

[46] D. H. Trivett, H. Pincon, and P. H. Rogers, J. Acoust. Soc. Am. 119, 3610 (2006).

[47] A. C. Hennion, and J. N. Decarpigny, J. Acoust. Soc. Am. 90, 3356 (1991).

[48] L. F. Shen, Z. Ye, and S. He, Phys. Rev. B. 68, 035109 (2003).

[49] H. Li, and M. Gupta, Fuzzy logic and intelligent systems (Boston, Kluwer Academic Publishers, 1995).

[50] H. J. Zimmermann, L. A. Zadeh, and B. R. Gaines, Fuzzy sets and decision analysis (Amsterdam, North-Holland, 1984).

[51] M. Mitchell, An Introduction to Genetic Algorithms (Cambridge, MIT Press, 1996). 
[52] Y. Xu et al, Chin. Phys. Lett. 22, 2557 (2005).

[53] Y. C. Chang, L. J. Yeh, and M. C. Chiu, Int. J. Numer. Meth. Engng. 62, 317 (2005). 


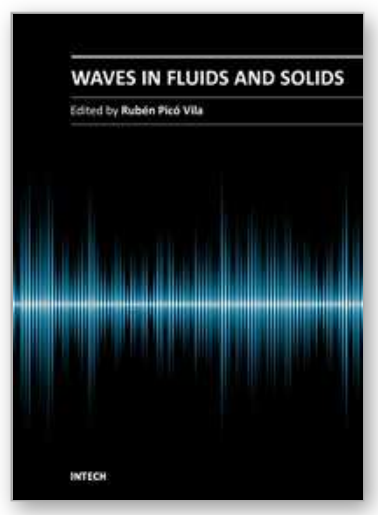

\author{
Waves in Fluids and Solids \\ Edited by Prof. Ruben Pico Vila
}

ISBN 978-953-307-285-2

Hard cover, 314 pages

Publisher InTech

Published online 22, September, 2011

Published in print edition September, 2011

Acoustics is an discipline that deals with many types of fields wave phenomena. Originally the field of Acoustics was consecrated to the sound, that is, the study of small pressure waves in air detected by the human ear. The scope of this field of physics has been extended to higher and lower frequencies and to higher intensity levels. Moreover, structural vibrations are also included in acoustics as a wave phenomena produced by elastic waves. This book is focused on acoustic waves in fluid media and elastic perturbations in heterogeneous media. Many different systems are analyzed in this book like layered media, solitons, piezoelectric substrates, crystalline systems, granular materials, interface waves, phononic crystals, acoustic levitation and soft media. Numerical methods are also presented as a fourth-order Runge-Kutta method and an inverse scattering method.

\title{
How to reference
}

In order to correctly reference this scholarly work, feel free to copy and paste the following:

Bin Liang, Ying Yuan, Xin-ye Zou and Jian-chun Cheng (2011). Acoustic Waves in Bubbly Soft Media, Waves in Fluids and Solids, Prof. Ruben Pico Vila (Ed.), ISBN: 978-953-307-285-2, InTech, Available from: http://www.intechopen.com/books/waves-in-fluids-and-solids/acoustic-waves-in-bubbly-soft-media

\section{INTECH}

open science | open minds

\section{InTech Europe}

University Campus STeP Ri

Slavka Krautzeka 83/A

51000 Rijeka, Croatia

Phone: +385 (51) 770447

Fax: +385 (51) 686166

www.intechopen.com

\section{InTech China}

Unit 405, Office Block, Hotel Equatorial Shanghai

No.65, Yan An Road (West), Shanghai, 200040, China

中国上海市延安西路65号上海国际贵都大饭店办公楼 405 单元

Phone: +86-21-62489820

Fax: +86-21-62489821 
(C) 2011 The Author(s). Licensee IntechOpen. This chapter is distributed under the terms of the Creative Commons Attribution-NonCommercialShareAlike-3.0 License, which permits use, distribution and reproduction for non-commercial purposes, provided the original is properly cited and derivative works building on this content are distributed under the same license. 